\title{
CHEMICGL REVIEWS
}

\section{X-ray and Neutron Scattering of Water}

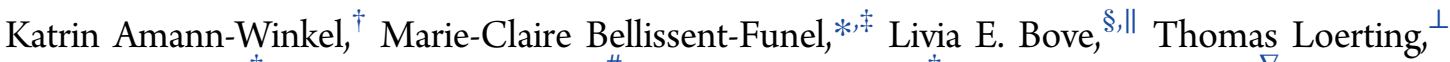
Anders Nilsson, ${ }^{\dagger}$ Alessandro Paciaroni, ${ }^{\#}$ Daniel Schlesinger, ${ }^{\dagger}$ and Lawrie Skinner ${ }^{\nabla}$

†Department of Physics, AlbaNova University Center, Stockholm University, SE-106 91, Stockholm, Sweden

${ }^{\ddagger}$ LLB, CEA, CNRS, Université Paris-Saclay, CEA Saclay, 91191 Gif-sur-Yvette, France

${ }^{\S}$ IMPMC, CNRS-UMR 7590, Université P\&M Curie, 75252 Paris, France

"Institute of Condensed Matter Physics, École Polytechnique Fédérale de Lausanne, CH-1015 Lausanne, Switzerland

${ }^{\perp}$ Institute of Physical Chemistry, University of Innsbruck, 6020 Innsbruck, Austria

\#Dipartimento di Fisica e Geologia, Università di Perugia, Via Alessandro Pascoli, I-06123 Perugia, Italy

${ }^{\nabla}$ Mineral Physics Institute, Stony Brook University, Stony Brook, New York 11794-2100, United States

ABSTRACT: This review article focuses on the most recent advances in X-ray and neutron scattering studies of water structure, from ambient temperature to the deeply supercooled and amorphous states, and of water diffusive and collective dynamics, in disparate thermodynamic conditions and environments. In particular, the ability to measure X-ray and neutron diffraction of water with unprecedented high accuracy in an extended range of momentum transfers has allowed the derivation of detailed $\mathrm{O}-\mathrm{O}$ pair correlation functions. A panorama of the diffusive dynamics of water in a wide range of

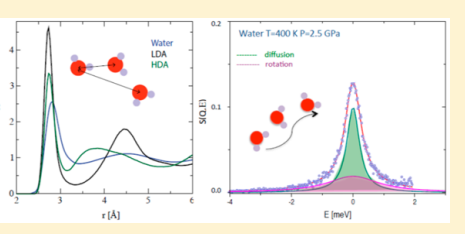
temperatures (from $400 \mathrm{~K}$ down to supercooled water) and pressures (from ambient up to multiple gigapascals) is presented. The recent results obtained by quasi-elastic neutron scattering under high pressure are compared with the existing data from nuclear magnetic resonance, dielectric and infrared measurements, and modeling. A detailed description of the vibrational dynamics of water as measured by inelastic neutron scattering is presented. The dependence of the water vibrational density of states on temperature and pressure, and in the presence of biological molecules, is discussed. Results about the collective dynamics of water and its dispersion curves as measured by coherent inelastic neutron scattering and inelastic X-ray scattering in different thermodynamic conditions are reported.

\section{CONTENTS}

1. Introduction

2. Water Structure from X-ray and Neutron Diffraction

2.1. Theory

2.2. Diffraction Measurements and Experimental Challenges

2.3. Neutron Diffraction Measurements of Water

2.4. X-ray Diffraction Measurements of Water

2.5. Isotope Effects on the Structure of Water

2.6. Structure of Water as a Function of Temperature: From the Liquid to the Amorphous Ices

2.6.1. Density Fluctuations (Small Angle Scattering)

2.6.2. Structure of Water in the Liquid State

2.6.3. Structure of Amorphous States of Water

3. Water Dynamics from Neutron Scattering

3.1. Diffusive Dynamics

3.2. Vibrational Dynamics

3.2.1. General Features

3.2.2. Intermolecular Vibrations

3.2.3. The Boson Peak

3.2.4. Intramolecular Vibrations

3.3. Collective Dynamics

3.3.1. General Features

\subsubsection{Fast Sound}

3.3.3. Low-Frequency Branch
7570

7571

7571

7572

7573

7574

7574

7575

7575

7576

7576

7578

7578

7580

7580

7581

7581

7582

7582

7582
4. Conclusion: Going Further into the Knowledge of the Structure and Dynamics of Water in Amorphous States and under Extreme Conditions of Temperature and Pressure

4.1. Improvement for the Structure of Water

Author Information

Corresponding Author $\quad 7583$

Author Contributions $\quad 7583$

Notes $\quad 7583$

Biographies $\quad 7584$

Acknowledgments $\quad 7584$

Abbreviations $\quad 7585$

$\begin{array}{ll}\text { References } & 7585\end{array}$

\section{INTRODUCTION}

Liquid water plays a central role in a wide range of scientific disciplines, such as physics, chemistry, geophysics, biology,

Special Issue: Water - The Most Anomalous Liquid

Received: November 10, 2015

Published: May 19, 2016 
atmospheric chemistry, food science, cryoscience, water treatment, electrocatalysis, and biomedicine. It is one of the main components that have allowed life on Earth to develop. Its presence on other planets is a topic of much general interest. Water is an ever-present material in our lives. The distribution of water around the globe influences weather on a daily basis, whereas on the longer time scale it has significantly shaped the geological and marine environment that we have today. Water has played and continues to play a highly significant role in the historical and political development of Man.

In a scientific context, water is a "simple" triatomic molecule with only two atomic components to constitute a hydride of oxygen, $\mathrm{OH}_{2}$. At room temperature water is a liquid, which distinguishes $\mathrm{H}_{2} \mathrm{O}$ from the hydrides of other elements close to oxygen in the periodic table, namely methane, ammonia, hydrogen sulfide, hydrogen chloride, and so on: these compounds are all gaseous at room temperature. At subzero temperature, water nucleates to form a solid that is less dense than the liquid phase. When subject to pressure and temperature variation water shows an amazing richness of polymorphs: 16 crystalline phases have so far been identified, as well as different amorphous forms. Water appears to have a number of unusual properties that account for its unique role in nature, and has frequently been termed as "anomalous". Most of water's "anomalies" derive from the presence of the hydrogen bond (HB), i.e. the electrostatic attraction between hydrogen atoms and the negatively charged region in the back of the oxygen site, and from the open, nearly tetrahedral, geometry of the molecule.

The anomalous physical properties of water become enhanced upon supercooling below the freezing point. Lowering the temperature, the strength of the hydrogen bond network increases and both cooperative effects between molecules and quantum effects become relevant. Of note is that, despite its rather high energy, the dynamics of the hydrogen bond is very rapid (of the order of $1 \mathrm{ps}$ ), and weakly affected by temperature ${ }^{1,2}$ and pressure ${ }^{3}$ variation, whereas the structural relaxation time increases by many orders of magnitude upon cooling. ${ }^{4}$ The delicate balance between this short lifetime and the existence of a HB network linking molecules together well above its percolation threshold is not yet fully unraveled. One of the keys to disclosing water's unique behavior thus resides in providing a coherent description of the hydrogen bond network structure and dynamics at the microscopic level on the broader range of thermodynamic conditions and environments.

In this review article, we focus on the most recent structural and dynamic properties of water in many different conditions of temperature and pressure as derived by $\mathrm{X}$-ray and neutron scattering experiments. These two techniques are complementary and well appropriate to probe these properties at a microscopic level and on the time scale of the hydrogen bond dynamics. The research described here has been performed at world leading research facilities including the neutron high flux reactor at the Institut Laue-Langevin (ILL) in Grenoble, the neutron spallation source ISIS in the U.K., the X-ray synchrotron radiation source at the Argonne National Laboratory (APS) in the United States, the European Synchroton Radiation Facility (ESRF) in Grenoble, and the DESY (Deutsches ElektronenSynchroton) in Hamburg.

Several review articles have reported on water structure and dynamics, ${ }^{5-10}$ but the recent advances in instrumentation for Xray diffraction both at synchrotron and Linac Coherent Light Source (LCLS) facilities, and for neutron diffusion at reactor sources and pulsed sources facilities, provide nowadays extremely accurate data on water structure, ${ }^{11}$ and gave access to previously unexplored states, ${ }^{12,13}$ thermodynamic conditions, ${ }^{12,14,3,15}$ and environments. ${ }^{16-18}$ In particular, the present article reviews the most recent X-ray and neutron diffraction results about the structure of liquid water and amorphous ice. We discuss wide angle scattering at ambient temperature on an extended $Q$-range and with an unprecedented low noise contamination, small angle scattering on ambient and supercooled water performed in a large Q-range with high accuracy and reproducibility, as well as neutron diffraction measurements on different amorphous ices produced by hyperquenching, vapor deposition, and pressure induced techniques. The translational and rotational diffusive dynamics of water under pressure of several gigapascals, as probed by quasi-elastic neutron scattering (QENS), is discussed in comparison with NMR, dielectric relaxation, and infrared spectroscopy. Just as at colder temperature, the anomalous behavior of water becomes more pronounced at higher pressure. Vibrational dynamics of bulk liquid water and hydration water from biomolecular systems as probed by inelastic neutron scattering and inelastic X-ray scattering is presented and compared with the results of molecular modeling.

\section{WATER STRUCTURE FROM X-RAY AND NEUTRON DIFFRACTION}

The microscopic structure of water is currently studied by using $\mathrm{X}$-ray and neutron diffraction techniques, which are complementary as differently sensitive to oxygen and hydrogen atoms, as described below. These diffraction techniques allow access to the intermolecular pair correlation function $g(r)^{19}$ of a system, i.e. the density probability of finding another atom lying in another molecule at a distance $r$ from any atom.

\subsection{Theory}

The basic theory for studies of X-ray and neutron scattering from molecular systems is well-known, ${ }^{19}$ and only the essentials will be reported here. These scattering techniques measure the differential scattering cross section $\mathrm{d} \sigma / \mathrm{d} \Omega$ that is essentially proportional to the structure factor $S_{\mathrm{M}}$ of a monatomic system or to a weighted sum of partial structure factors for a polyatomic system.

The differential scattering cross section for scattering of neutrons or X-rays by an assembly of fixed nuclei (forming molecules or not) is given in the static approximation by

$$
\frac{\mathrm{d} \sigma}{\mathrm{d} \Omega}(\mathbf{Q})=\left\langle\sum_{i j} a_{i} a_{j} \exp \left[i \mathbf{Q} \cdot \mathbf{r}_{i j}\right]\right\rangle+\sum_{i j} \frac{\sigma_{j}}{4 \pi}
$$

where $a_{i}$ is the scattering factor of atom $i$ (for neutrons, $a_{i}=\left\langle b_{i}\right\rangle$ is the coherent scattering length of the $i$ nucleus; for X-rays, $a_{i}=f_{i}$ is the atomic scattering factor).

In the case of neutrons: we define the coherent and incoherent scattering lengths of the nucleus after averaging over all isotopes and nuclear spins:

$$
\begin{aligned}
& b_{\text {coh }, i}=\left\langle b_{i}\right\rangle \\
& b_{\text {incoh }, i}=\left[\left\langle b_{i}^{2}\right\rangle-\left\langle b_{i}\right\rangle^{2}\right]^{1 / 2}
\end{aligned}
$$

and the incoherent scattering cross sections $\sigma_{\text {incoh }, i}=4 \pi\left(b_{\text {incoh }, i}\right)^{2}$. This is simply the average and standard deviation of the sample's scattering length distribution respectively.

$Q=4 \pi \sin \theta / \lambda$ is the modulus of the scattering wave vector in the case of elastic scattering where $2 \theta$ is the diffraction angle and $\lambda$ is the wavelength of the radiation; the vector $r_{i j}=r_{i}-r_{j}$ gives the 
relative position of scattering centers $i$ and $j$. The broken brackets in eq 1 denote an average over the grand-canonical ensemble

The differential scattering cross section (eq 1) can be split into a self-part $(i=j)$ and a distinct part $(i \neq j)$ :

$$
\mathrm{d} \sigma / \mathrm{d} \Omega=(\mathrm{d} \sigma / \mathrm{d} \Omega)^{\text {self }}+(\mathrm{d} \sigma / \mathrm{d} \Omega)^{\text {distinct }}
$$

The self-part itself can be split into an incoherent and a selfcoherent part

$$
(\mathrm{d} \sigma / \mathrm{d} \Omega)^{\text {self }}=(\mathrm{d} \sigma / \mathrm{d} \Omega)_{\text {coh }}^{\text {self }}+(\mathrm{d} \sigma / \mathrm{d} \Omega)_{\text {incoh }}^{\text {self }}
$$

The distinct part can be separated into intra- and intermolecular parts corresponding to correlations between atoms within the same molecule and atoms belonging to different molecules, respectively:

$$
(\mathrm{d} \sigma / \mathrm{d} \Omega)^{\text {distinct }}=(\mathrm{d} \sigma / \mathrm{d} \Omega)^{\text {intra }}+(\mathrm{d} \sigma / \mathrm{d} \Omega)^{\text {inter }}
$$

The differential scattering cross section may thus be expressed by the relation

$$
\begin{aligned}
& \mathrm{d} \sigma / \mathrm{d} \Omega(Q)=(\mathrm{d} \sigma / \mathrm{d} \Omega)^{\text {self }}(Q)+(\mathrm{d} \sigma / \mathrm{d} \Omega)^{\text {intra }}(Q) \\
& \quad+(\mathrm{d} \sigma / \mathrm{d} \Omega)^{\text {inter }}(Q)
\end{aligned}
$$

The structure factor of a molecular liquid $S_{\mathrm{M}}(Q)$ is defined from the distinct part of the differential scattering cross section. For a molecular liquid, it may be split into two parts:

$$
S_{\mathrm{M}}(Q)=f_{1}(Q)+D_{\mathrm{M}}(Q)
$$

$f_{1}(Q)$ is the molecular form factor and the $D_{M}(Q)$ function contains all the intermolecular contributions.

For $Q \rightarrow 0, \lim _{Q \rightarrow 0}\left(S_{\mathrm{M}}\right)=\rho k_{\mathrm{B}} T \chi_{\mathrm{T}}$, where $\rho$ is the molecular number density, $k_{\mathrm{B}}$ is the Boltzmann constant, $T$ is the absolute temperature and $\chi_{T}$ is the isothermal compressibility.

$S_{\mathrm{M}}(\infty)=\left(\sum b_{i, \mathrm{coh}}^{2}\right) /\left(\sum b_{i, \mathrm{coh}}\right)^{2}$ is the asymptotic value of $S_{\mathrm{M}}$ at large $Q$. For a molecular liquid it is important to remove the intramolecular contribution $f_{1}(Q)$ from the structure factor $S_{\mathrm{M}}(Q)$ in order to access only the intermolecular contribution $D_{\mathrm{M}}(Q)$. By Fourier transformation, one can calculate the pair correlation function $g(r)$

$$
g(r)=1 /\left(2 \pi^{2} \rho r\right) \int_{0}^{\infty} Q D_{\mathrm{M}}(Q) \sin (Q r) \mathrm{d} Q+1
$$

for the intermolecular terms only. The function $g(r)$ is a combination of the different partial correlation functions.

\subsection{Diffraction Measurements and Experimental Challenges}

X-ray diffraction studies of liquid water, which date back to the 1930s, give pair distribution functions $g(r)$ with contributions mainly from oxygen-oxygen $(\mathrm{O}-\mathrm{O})$ correlations, $g_{\mathrm{OO}}(r)$, and oxygen-hydrogen $(\mathrm{O}-\mathrm{H})$ correlations, $g_{\mathrm{OH}}(r)$, while the hydrogen-hydrogen $(\mathrm{H}-\mathrm{H})$ correlations, $g_{\mathrm{HH}}(r)$ give negligible contributions, as discussed below. The dominating $\mathrm{O}-\mathrm{O}$ contribution exhibits peaks at 2.8 and $4.5 \AA^{20}$ Although these peak positions have a ratio close to the 1.633 expected for a tetrahedral $\mathrm{O}-\mathrm{O}-\mathrm{O}$ angle, they are found to be broad and overlapping in ambient liquid water, leaving a wide range of $\mathrm{O}-$ $\mathrm{O}-\mathrm{O}$ angles, rather than a well-defined tetrahedral arrangement. The precise degree of tetrahedrality in ambient water thus remains a subject of debate, ${ }^{5,21-23}$ and even though it can be easily quantified in atomistic models, the correspondence of models to actual water is often imperfect. It is clear, however, that liquid water is more disordered than the ideal tetrahedral arrangements of amorphous $\mathrm{Si}$ and low-density amorphous ice.
Unlike ambient liquid water, these systems have well-defined first peaks in their measured $g(r)$ patterns, and an average number of nearest neighbors very close to 4.0. Though the four-coordinated motif is still the dominant one in water, a significant amount of three-coordinated motifs are present, consistent with the trigonal geometry of water electron density, as well as a large number of bifurcated interactions ${ }^{24}$ accounted for by five neighbors, and which play a fundamental role in the diffusion mechanism. ${ }^{25}$

While X-ray diffraction patterns of water contain very little $\mathrm{H}-$ $\mathrm{H}$ information (the hydrogen atom only has one electron and its $\mathrm{X}$-ray scattering cross section is thus very small), neutron diffraction measurements on $\mathrm{H}_{2} \mathrm{O}-\mathrm{D}_{2} \mathrm{O}$ mixtures can provide separated partial pair distribution functions $\left(g_{\mathrm{OO}}(r), g_{\mathrm{OH}}(r)\right.$, and $g_{\mathrm{HH}}(r)$ assuming $\mathrm{H}-\mathrm{D}$ equivalence). An early measurement of these $g_{\alpha \beta}(r)$ functions by Narten et al. ${ }^{26}$ is shown in Figure 1. These three $g_{\alpha \beta}(r)$ patterns, and later improved measurements, provide detailed local structure information and a reference for models of liquid water.

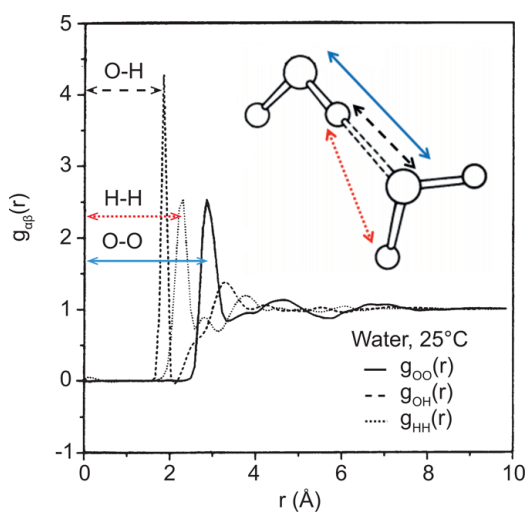

Figure 1. Partial pair distribution functions obtained from neutron diffraction and $\mathrm{H}-\mathrm{D}$ substitution. Reprinted with permission from ref 26. Copyright 1982 American Association for the Advancement of Science.

Precise partial $g_{\alpha \beta}(r)$ measurements, however, are often hard to achieve. First, three or more $S(Q)$ measurements must be decomposed into the three partial structure factors, and then these decomposed functions are Fourier transformed to obtain the $g_{\alpha \beta}(r)$ patterns. When obtaining $g_{\alpha \beta}(r)$ functions, errors are often hard to track. Noise and systematic errors in $S(Q)$ can appear in the $g(r)$ as unphysical oscillations and erroneous peaks. Hence precise measurements are required to obtain meaningful results, yet water poses added technical difficulties: $\mathrm{X}$-rays scatter very weakly because of water's low electron density, whereas the large incoherent neutron scattering cross section and low mass of $\mathrm{H}$ lead to large attenuation, multiple scattering, and recoil corrections in neutron diffraction. The method of $\mathrm{H}-\mathrm{D}$ substitution is also a bad case for isotope equivalence, resulting in $\mathrm{H}_{2} \mathrm{O}$ and $\mathrm{D}_{2} \mathrm{O}$ having slightly different structures and properties; for example, the $\mathrm{D}_{2} \mathrm{O}$ temperature of maximum density is $7.2 \mathrm{~K}$ higher than that of $\mathrm{H}_{2} \mathrm{O}$. Several different strategies for minimizing these problems are used in the literature, and inconsistencies have frequently led to disagreement. The different $g_{\alpha \beta}(r)$ contributions are shown in Figure 1. The more pronounced features of the $g_{\alpha \beta}(r)$ patterns are broadly correct, but as noted by the original authors, there are significant errors present. These errors make the smaller features, such as the peak in $g_{\mathrm{HH}}(r) \sim 2.8 \AA \AA$, unreliable. 
Recently, the $\mathrm{O}-\mathrm{O}$ distribution function $g_{\mathrm{OO}}(r)$ has been determined to greatly improved accuracy from both neutron ${ }^{27}$ and X-ray diffraction ${ }^{11}$ that agree almost quantitatively.

\subsection{Neutron Diffraction Measurements of Water}

Neutrons see nuclei, rather than the diffuse electron cloud seen by X-rays. This has the major advantage of allowing sensitivity to light atoms such as the hydrogens of water. A key property of neutron diffraction is that the coherent scattering length $b_{c}$ depends on the isotope. The accepted coherent scattering lengths $\left(b_{c}\right)$ for $\mathrm{O}$ and $\mathrm{H}$ (data from ref 28 , with the exception ${ }^{29}$ of ${ }^{18} \mathrm{O}$ ) given in Table 1 demonstrate the large difference between ${ }^{1} \mathrm{H}$ and $\mathrm{D}\left({ }^{2} \mathrm{H}\right)$, as well as the small, but usable difference between ${ }^{16} \mathrm{O}$ and ${ }^{18} \mathrm{O}$.

Table 1. Key Neutron Scattering Parameters for Water Components (Coherent Scattering Length $b_{\mathrm{c}}$ and Incoherent Scattering Cross Section $\sigma_{\text {inc }}{ }^{a}$ )

$\begin{array}{lcc}\text { isotope } & b_{\mathrm{c}}{ }^{28}(\mathrm{fm}) & \sigma_{\text {inc }}{ }^{28}(\mathrm{barn}) \\ { }^{1} \mathrm{H} & -3.742 & 80.27 \\ { }^{2} \mathrm{H}(\mathrm{D}) & 6.674 & 2.05 \\ { }^{16} \mathrm{O} & 5.805 & 0 \\ { }^{18} \mathrm{O} & 6.009^{29} & 0\end{array}$

${ }^{a}$ Note the very large $\sigma_{\text {inc }}$ of ${ }^{1} \mathrm{H}$ (problematic), and the large difference between $b_{\mathrm{c}}$ values of ${ }^{1} \mathrm{H}$ and $\mathrm{D}$ (advantageous).

Before accessing the differential scattering cross section or the structure factor of water, corrections to the experimental data have to be done. Neutron diffraction correction procedures for water have been described in detail many times..$^{27,30,31}$ Here we give an overview of the major problems and approaches taken to achieve optimum measurements.

A major problem for water measurements is the large incoherent scattering cross section $\sigma_{\text {ino }}$ which does not contain any structural information, but increases the multiple scattering and attenuation effects. To reduce these multiple scattering and attenuation effects arising from the large $\sigma_{\text {inc }}$ of $\mathrm{H}_{2} \mathrm{O}$, it is often preferable to use $\mathrm{H}-\mathrm{D}$ mixtures with less than $50 \% \mathrm{H}_{2} \mathrm{O} .{ }^{31}$ Also, since the $\mathrm{H}-\mathrm{D}$ isotope effect is very similar to a temperature shift, ${ }^{32}$ the isotope equivalence can be improved by measuring the different $\mathrm{H}-\mathrm{D}$ mixtures at different temperatures, using the offsets determined by X-ray diffraction as a guide. ${ }^{31-33}$ This effect is expected to become significant in the supercooled regime where isotope effects approximate a temperature shift of about $\sim 10 \mathrm{~K}^{33}$ By contrast, the isotope effect in crystalline ices ${ }^{34}$ is much smaller than in the supercooled liquid. Melting temperatures, polymorphic transformation temperatures and triple points deviate by only $\sim 2-5 \mathrm{~K}$ upon $\mathrm{H}-\mathrm{D}$ substitution. ${ }^{31}$

A second problem is the Placzek falloff, arising from large inelasticity effects due to the near-equal mass of $\mathrm{H}$ nuclei and incident neutrons. In general the ratio of low- $Q$ to high- $Q$ scattering levels from this falloff is approximately $(A+1)^{2} / A^{2}$, where $A$ is the atomic mass. This shows that the falloff for ${ }^{1} \mathrm{H}$ is a factor of 4 , while for $\mathrm{D}$ it is 2.5 , and for ${ }^{16} \mathrm{O}$ the factor is only 1.13 . Figure 2 shows the total differential scattering cross sections measured using different techniques showing the severity of the Placzek falloff for different measurement setups. Since the Placzek falloff depends most strongly on scattering angle $(2 \theta)$, one can reduce the slope over a given $Q$-range $(Q=4 \pi \sin (\theta) / \lambda)$ by using a constant $\theta$, and varying $\lambda$. This is typically achieved using time-of-flight (TOF) neutron scattering at a pulsed neutron source (Figure 2, right). The use of high-energy neutrons in TOF measurements also reduces multiple scattering and attenuation effects. In this method the wavelength of scattered neutrons is assigned according to their arrival time. TOF neutron measurements also incur technical difficulties: the wide range of wavelengths requires detailed analysis and modeling of effects such as multiple scattering, Placzek, and sample attenuation corrections. Although we call the Placzek a falloff, for TOF neutron measurements the instrument geometry can result in this correction taking different shapes.
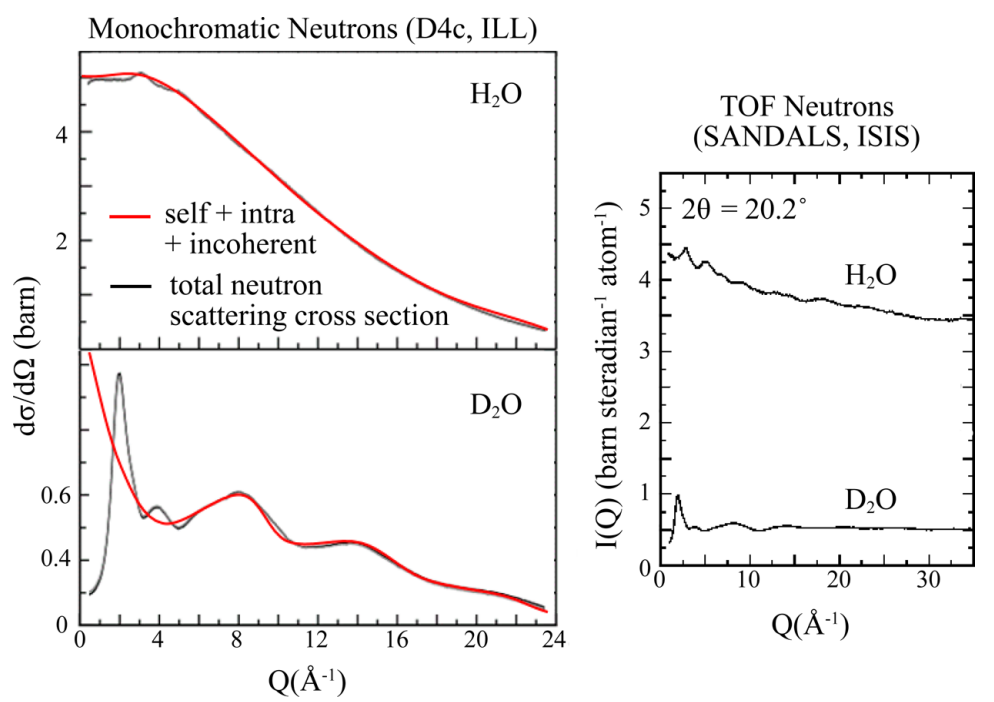

Figure 2. Total differential scattering cross sections of pure $\mathrm{H}_{2} \mathrm{O}$ and pure $\mathrm{D}_{2} \mathrm{O}$ water as measured by two different neutron scattering techniques. (left) Monochromatic neutrons $(\lambda=0.5 \AA)$ using the reactor source ILL (gray lines). The red line corresponds to the sum of self plus intramolecular plus incoherent contributions (see section 2.1 for details). (right) Time-of-flight neutron diffraction (TOF) at the pulsed neutron source ISIS, which uses a wide band of incident neutron energies $(\lambda \sim 0.1-3 \AA$ ) (black lines). Reprinted with permission from ref 36 . Copyright 2012 IOP Publishing. Reprinted with permission from ref 30. Copyright 2006 IOP Publishing. 
Alternatively monochromatic neutron experiments, using a reactor source, vary only $\theta$ to collect a sufficiently wide $Q$-range (Figure 2, left). In this case Placzek falloff for $\mathrm{H}_{2} \mathrm{O}$ is large and problematic. One method for minimizing this problem is to use $\mathrm{H}_{2}{ }^{16} \mathrm{O}$ and $\mathrm{H}_{2}{ }^{18} \mathrm{O}$ differences, where the $\mathrm{H}-\mathrm{D}$ content is identical in each sample; then only a small residual oxygen Placzek slope remains in the difference between the two measurements, while partial structure factor information can also be obtained. ${ }^{35,36}$

\subsection{X-ray Diffraction Measurements of Water}

$\mathrm{X}$-ray scattering of water is caused by interactions of the incident $\mathrm{X}$-ray photons with the electron density in the water molecule located mostly at the oxygen atom. The coherent part of the Xray diffraction signal is thus dominated by oxygen-oxygen correlations. This is in contrast to neutron diffraction measurements, which are sensitive to scattering from both hydrogen (deuterium) and oxygen atoms. While $\mathrm{D}_{2} \mathrm{O}$ is a relatively strong coherent neutron scatterer it is a weak X-ray scatterer; on top of this, much of the measured scattered X-ray signal is either selfscattering or Compton scattering, leaving an extremely small coherent signal, as illustrated in Figure 3.

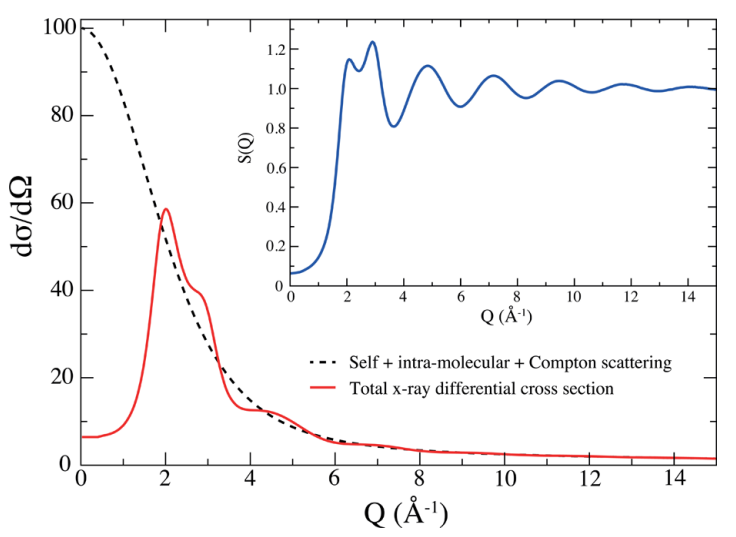

Figure 3. Total X-ray differential scattering cross section $\mathrm{d} \sigma / \mathrm{d} \Omega(Q)$ of water $^{11}$ (solid red line). The sum of self-scattering, intramolecular scattering, and Compton scattering is indicated by the dashed black line. The total coherent X-ray scattering cross section, called the total structure factor $S(Q)$ (inset, blue line), is obtained after subtraction of the self, intramolecular, and Compton scattering contributions from the total X-ray differential scattering cross section and after normalization as described in the text. The data presented here were taken from ref 11.

However, an advantage of X-ray scattering compared to neutron scattering is the availability of large monochromatic flux at short wavelengths $(\sim 0.1 \AA)$; this allows angular corrections and statistical noise to be minimized in the measurements.

Different approaches have been used in the literature to extract the X-ray structure factor quantifying the coherent intermolecular scattering from X-ray diffraction measurements, and detailed comparisons of these schemes have been performed. ${ }^{37,11}$ In the independent atom approximation (IAA), the concentration-weighted sum of the scattering intensity of the individual atoms is subtracted from the total scattering intensity. This atomic normalization scheme has been improved by the use of modified atomic form factors (MAFFs), $f_{\alpha}$, which take charge redistributions between the atoms within a water molecule and valence-electron delocalization due to chemical bonding into account. $^{38}$
In the molecular normalization scheme, on the other hand, the square of the molecular form factor (MFF), here denoted $C(Q)$, is subtracted from the scattering intensity. The MFF can be obtained from quantum calculations. ${ }^{39}$ The resulting coherent intermolecular scattering intensity is commonly normalized by $B(Q)=\left(\sum_{\alpha} c_{\alpha} f_{\alpha}(Q)\right)^{2}$ and the square of the number of atoms per molecule ( 9 in the case of water), and the total X-ray structure factor $S(Q)$ can thus be calculated:

$$
S(Q)-1=(I(Q)-C(Q)) /(9 B(Q))
$$

The total structure factor mainly consists of contributions from $\mathrm{O}-\mathrm{O}$ correlations, with a small contribution from $\mathrm{O}-\mathrm{H}$ correlations and a negligible contribution from $\mathrm{H}-\mathrm{H}$ correlations $\left(\sim 1 \%\right.$ at $Q<5 \AA^{-1}$ and smaller elsewhere). The $\mathrm{O}-\mathrm{O}$ partial structure factor can then be calculated by subtracting the intermolecular $\mathrm{O}-\mathrm{H}$ contribution, which can be obtained from molecular dynamics simulations, combined X-ray and neutron diffraction analyses, or, as recently demonstrated, from oxygen isotope substituted neutron diffraction measurements. ${ }^{35^{\circ}}$ As discussed already in section 2.1, the real space molecular distribution can be obtained by Fourier transformation of the structure factor. For a detailed description of X-ray scattering analysis and correction procedures see refs 11 and 40.

\subsection{Isotope Effects on the Structure of Water}

While the $\mathrm{H}-\mathrm{D}$ substitution neutron diffraction technique typically assumes structural equivalence between $\mathrm{H}_{2} \mathrm{O}$ and $\mathrm{D}_{2} \mathrm{O}$, $\mathrm{X}$-ray diffraction offers the opportunity to investigate their differences. Since isotopes have the same electron number, differences observed with $\mathrm{X}$-ray diffraction between $\mathrm{H}_{2}{ }^{16} \mathrm{O}$, $\mathrm{D}_{2}{ }^{16} \mathrm{O}, \mathrm{H}_{2}{ }^{18} \mathrm{O}$, and $\mathrm{D}_{2}{ }^{18} \mathrm{O}$ correspond directly to the nonequivalence of their structures. Such isotope difference measurements find that the $\mathrm{H}_{2} \mathrm{O}-\mathrm{D}_{2} \mathrm{O}$ X-ray difference is very similar to a temperature shift of $5-10 \mathrm{~K}$, and that the difference increases with decreasing temperature. ${ }^{32}$ The $\mathrm{H}_{2}{ }^{18} \mathrm{O}-\mathrm{H}_{2}{ }^{16} \mathrm{O}$ X-ray structure differences, however, are roughly an order of magnitude smaller than the $\mathrm{H}_{2} \mathrm{O}-\mathrm{D}_{2} \mathrm{O}$ difference.

A limitation of X-ray isotope difference measurements is that they are mainly sensitive to the differences in the $\mathrm{O}-\mathrm{O}$ correlations $\left(g_{\mathrm{OO}}(r)\right)$. Neutron scattering measurements, however, are in principle able to give information on all three $(\mathrm{O}-\mathrm{O}$, $\mathrm{O}-\mathrm{H}$, and $\mathrm{H}-\mathrm{H}$ ) partial differences from isotope effects. This separation requires very high precision measurements, at several different concentration $\mathrm{H}-\mathrm{D}$ mixtures, and/or resolving the very small scattering contrast between ${ }^{16} \mathrm{O}$ and ${ }^{18} \mathrm{O}$ enriched water. $^{35,41}$

Recent investigations about the isotope effect on the structure of liquid water have been focused on the $r_{\mathrm{OD}}$ and $r_{\mathrm{OH}}$ intramolecular bond lengths. When measured with $\mathrm{H}-\mathrm{D}$ substitution, a $3 \%$ difference is observed ${ }^{42}$ in time-of-flight neutron diffraction measurements at small angles, leading the authors to concur with an earlier X-ray Raman spectroscopy study $^{43}$ which concluded an enhanced hydrogen bond asymmetry in $\mathrm{H}_{2} \mathrm{O}$ in addition to the temperature offset reported by Hart et al. ${ }^{32}$ Oxygen isotope substitution leads to smaller effects where, e.g., monochromatic neutron diffraction measurements using ${ }^{18} \mathrm{O}-{ }^{16} \mathrm{O}$ differences find $r_{\mathrm{OD}}=0.985 \AA$ and $r_{\mathrm{OD}}=$ $0.990 \AA$, a $0.5 \%$ difference. ${ }^{35,36}$ At least part of the difference in results arises from the small size of the isotope effect and the large corrections required of the data. The determination of the $\mathrm{H}-\mathrm{H}$ and $\mathrm{O}-\mathrm{O}$ intermolecular isotope effects, especially at deep supercooling, is far from being achieved and would be of particular interest in future investigations. 
2.6. Structure of Water as a Function of Temperature: From the Liquid to the Amorphous Ices

2.6.1. Density Fluctuations (Small Angle Scattering). Small-angle X-ray scattering (SAXS) and small-angle neutron scattering (SANS) are the most direct probes of density fluctuations on different length scales in a single component liquid. Through an enhancement of the structure factor at low momentum transfer, $Q$ small deviations from the average electron density at different length scales can be reliably identified. ${ }^{44}$ Previous SAXS studies of water have mostly focused on the supercooled region and given contradictory results, reporting both positive $e^{45-47}$ and zero enhancement ${ }^{48,49}$ at low $Q$. With the development of third-generation synchrotron light sources, the ability to perform SAXS has been greatly advanced and measurements can now be performed in a large Q-range with high accuracy and reproducibility. ${ }^{50}$

Figure 4 depicts the scattering structure factor, $S(Q)$, at different temperatures varying from 347 to $280 \mathrm{~K}^{51}$ and from 284
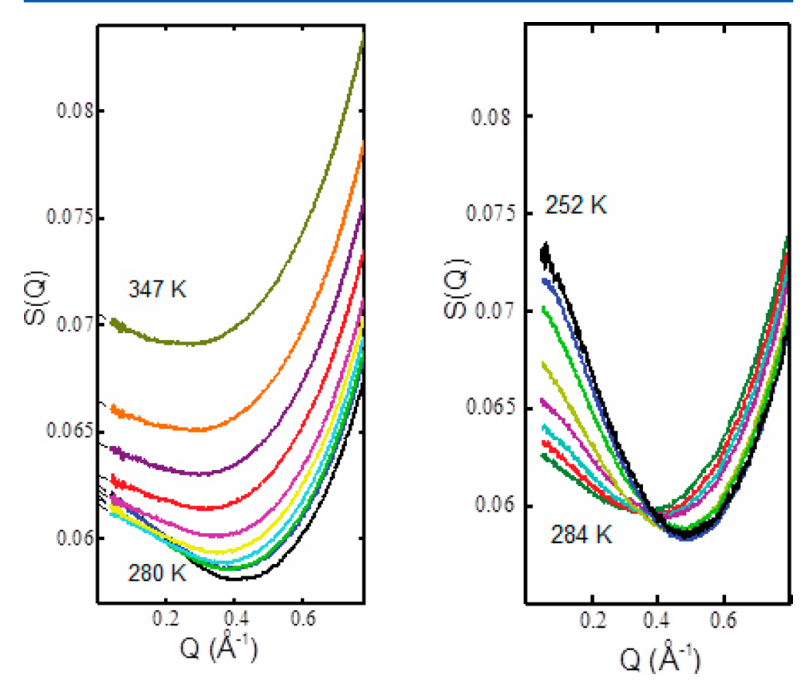

Figure 4. Experimental structure factor, $S(Q)$, derived from SAXS of $\mathrm{H}_{2} \mathrm{O}$ : (left, bottom to top at high $Q$ ) at $280,284,289,293,298,302,311$, 320,329 , and $347 \mathrm{~K}_{;}^{51}$ (right, top to bottom at low $Q$ ) at $252,254,258$, $263,268,273,278$, and $284 \mathrm{~K}^{52}$ Reprinted with permission from ref 51. Copyright 2009 National Academy of Sciences. Reprinted with permission from ref 52. Copyright 2010 American Institute of Physics Publishing LLC.

$\mathrm{K}$ down to $252 \mathrm{~K}^{52} \mathrm{~S}(\mathrm{Q})$ shows an enhanced scattering as $Q$ approaches zero where the slope of the curve changes from positive to increasingly negative and with a higher intercept with decreasing temperatures, indicating density fluctuations in the system which increase at low temperatures. This is different behavior in comparison to a simple liquid such as ethanol where instead the temperature variation is a parallel shift and there is no enhancement at low $Q .{ }^{53}$

There is a thermodynamic relationship that relates the isothermal compressibility $\chi_{T}$ to the structure factor at $Q=0$ as $S(0)=\rho k_{\mathrm{B}} T \chi_{T}$ (see section 2.1). ${ }^{54}$ Figure 5 compares the isothermal compressibility determined from measurements of the velocity of sound in water ${ }^{55}$ and as obtained from the SAXS data ${ }^{51,52}$ which demonstrates an excellent agreement. This shows that the anomaly of the minimum and then the rise of $\chi_{T}$ toward cooler temperatures is directly related to the enhancement observed in the SAXS data and thus to the density inhomogeneity arising due to density fluctuations. ${ }^{56}$ This has been interpreted in terms of fluctuations between spatially separated structural components of high-density-liquid (HDL) and low-density-liquid (LDL) local environments. ${ }^{51-53,57}$

This interpretation of the SAXS data has generated a highpitched debate, ${ }^{22,60-62}$ which is natural since there is no formally strict way to divide the enhancement in the SAXS data at low $Q$ into different contributions to derive the correlation length. Therefore the interpretation of the experimental data is subject to differences in opinion, however with the constraint that the interpretation should be consistent with, and build on, the existing data. Recent ${ }^{59}$ theoretical simulations using the TIP4P/ 2005 model have provided additional insights into the low $Q$ enhancement since this model gives some qualitative agreement with the anomalous rise in $\chi_{T}$ at temperatures below the minimum. Figure 5 (right) shows how the low $Q$ signal could be divided into partial structure factors and that the enhancement comes from structural fluctuations of different local hydrogen bond configurations due to attractive interactions of molecules in similar environments. ${ }^{58}$ Figure 5 (left, inset) shows colored regions in a simulation box of 45000 molecules at $253 \mathrm{~K}$ of TIP4P $/ 2005^{63}$ water where two different selection criteria have been used of either high tetrahedrality (blue) corresponding to LDL or high density (yellow) as HDL, and indeed we observe a spatial separation. ${ }^{59}$ We clearly see the inhomogeneous structure where the regions of LDL and HDL occupy different parts in the real space.

There is a temperature-dependent size distribution in terms of the number of water molecules contributing to the tetrahedral patches giving an asymptotic contribution $\left(g^{\mathrm{A}}(r) \sim \exp (-r / \xi) /\right.$ $r)(r \gg 1))$ to the pair correlation function, with $\xi$ being the correlation length. The correlation length $\xi$ should not be mistaken for a specific length in the liquid but represents the damping factor of the long-range enhancement of the pair correlation function. It is clear that the extracted correlation lengths are small, $2-3 \AA$, so how can this be understood and related to the real-space correlation? Is such a small correlation length, comparable to molecular dimensions, even relevant?

The same anomalous component in the SAXS data can, however, be analyzed in different ways depending on the expected character of the system. In terms of critical behavior the fit with an asymptotic exponential decay of the correlation function is the established approach, but when dealing with, e.g., macromolecules or colloids with fixed structure, the same curve shape would rather be analyzed in terms of a Guinier analysis ${ }^{64}$ with the low- $Q$ shape described by the scattering expression $\exp \left(-\frac{1}{3} R_{\mathrm{G}}{ }^{2} Q^{2}\right)$, where $R_{\mathrm{G}}$ is the Guinier radius, which, in contrast to the $\xi$ correlation length, in its interpretation is more directly related to real-space physical dimensions. From the scattering expression one finds $R_{\mathrm{G}}=\xi \sqrt{3}$ and, assuming a spherical shape, the diameter becomes $D=2 \sqrt{3 / 5} R_{G}$, which with $\xi=2-3 \AA$ gives $D=8-12 \AA^{51}$ However, we have to be aware that there will be many sizes, and the derived value of $D$ only gives a rough estimation of the mean value of the distribution. If the sizes were much smaller than $10 \AA$, we would not detect any enhancement at all, and if they were much larger, the enhancement would be many orders of magnitude larger and the correlation length much longer. Although we lack information on the time scale of the fluctuations, the attosecond interaction time of the X-ray scattering process, compared with picoseconds for $\mathrm{H}$-bond dynamics, allows considering the SAXS data as an instantaneous snapshot of the structure. 

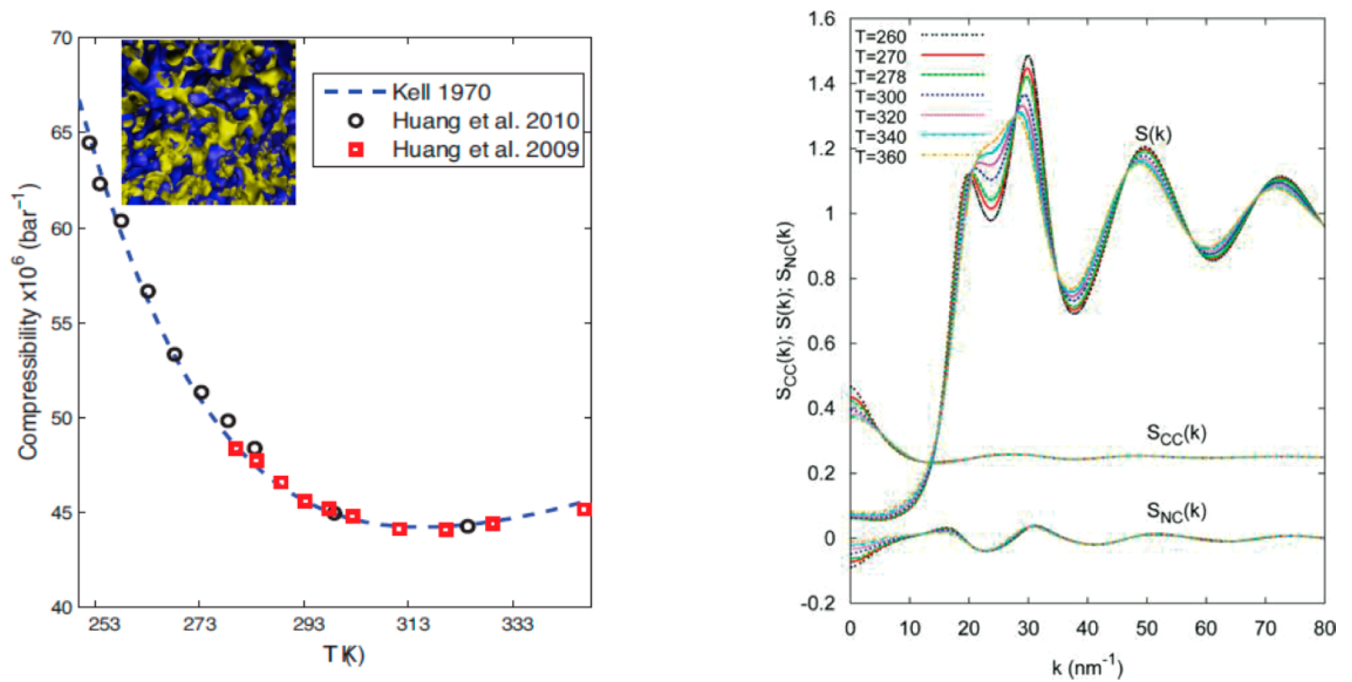

Figure 5. (left) Isothermal compressibility determined from either macroscopic thermodynamic measurements (dashed line) ${ }^{55}$ or extracted from the SAXS data (circles and squares) ${ }_{51,52}$ (right) Concentration-concentration $\left(S_{\mathrm{CC}}(k)\right)$, density-density $(S(k))$ ), and density-concentration $\left(S_{\mathrm{NC}}(k)\right){ }^{58}$ (inset) Isosurfaces at $253 \mathrm{~K}$ of high-density fields (yellow) and high tetrahedrality (blue) from TIP4P/2005 simulations. ${ }^{59}$ The length of the box is $\sim 106$ Å. Reprinted with permission from ref 51. Copyright 2009 National Academy of Sciences. Reprinted with permission from ref 52. Copyright 2010 American Institute of Physics Publishing LLC.

2.6.2. Structure of Water in the Liquid State. Two recent experimental studies ${ }^{11,27}$ provide precise $\mathrm{O}-\mathrm{O}$ pair distribution functions of liquid water at ambient conditions, including error bars. While the former uses a wide number of data sets and empirical potential structure refinement (EPSR) modeling to obtain all three partial pair distribution functions, the latter uses only diffraction data to provide a model-independent determination of the $\mathrm{O}-\mathrm{O}$ pair distribution function. Although these two studies are largely independent, it should be noted that an early version of the data in ref 11 is used as one of the inputs into the structure models in ref 27.

These recent studies are in close agreement (see Figure 6), though not fully within each other's error bars. Table 2 compares the $\mathrm{O}-\mathrm{O}$ coordination numbers, $n_{\mathrm{OO}}(r)=4 \pi \rho \int_{0}^{r} g_{\mathrm{OO}}\left(r^{\prime}\right) r^{\prime 2} \mathrm{~d} r^{\prime}$, depending on the cutoff $r$, and the first peak heights, $g_{1}$, in the $\mathrm{O}-$ $\mathrm{O}$ pair distribution function $g_{\mathrm{OO}}(r)$ among three recent studies. ${ }^{11,27,36}$ The Soper and Zeidler et al. $g_{\mathrm{OO}}(r)$ results $^{27,36}$ give a very slightly higher $\mathrm{O}-\mathrm{O}$ coordination and a very slightly lower first $\mathrm{O}-\mathrm{O}$ peak height $\left(g_{1}\right)$ than the Skinner et al. $g_{\mathrm{OO}}(r)$ determination. ${ }^{11}$ This may be because the Skinner et al. ${ }^{11}$ result is mainly from $\mathrm{X}$-ray diffraction on $\mathrm{H}_{2} \mathrm{O}$ while the other two studies use mainly neutron diffraction from $\mathrm{D}_{2} \mathrm{O}-\mathrm{H}_{2} \mathrm{O}$ mixtures.

Figure 6A shows $g_{\mathrm{OO}}(r)$ values from two different experiments. ${ }^{11,27}$ These measurements are in good agreement within the error bars established in ref 11 but differ slightly around the positions of the first peak and the first minimum, as indicated. The temperature dependence of water's $g_{\mathrm{OO}}(r)$ has also been studied recently with improved accuracy. ${ }^{40}$ In this study X-ray measurements were made over a wide temperature range, from which the $g_{\mathrm{OO}}(r)$ and $r d_{\mathrm{OO}}(r)$ pair distribution functions were extracted. In Figure 6B, the $r d_{\mathrm{OO}}(r)$ data are shown for different temperatures, color coded from the coldest measured temperature at $254.2 \mathrm{~K}$ (black) to the hottest measured temperature at $365.9 \mathrm{~K}$ (red). Skinner et al. ${ }^{40}$ find that the average number of $\mathrm{O}-\mathrm{O}$ neighbors up to $3.3 \AA$ is almost constant at $4.3 \pm 0.1$ over this wide temperature range. As temperature is increased, however, shorter more tightly bound neighbors are exchanged for longer, less well-bound neighbors (Figure 6B). These new measurements also resolve detailed structural changes out to $\sim 15$ $\AA$. From this data, the second peak position in the X-ray structure factor $S_{\mathrm{X}}(Q)$ prior to $\mathrm{O}-\mathrm{H}$ subtraction and Fourier transformation $\left(Q_{2}\right)$ and the second peak position $\left(r_{2}\right)$ in $g_{\mathrm{OO}}(r)$ are observed to both change their slope (temperature derivative) around the temperature of the compressibility minimum ( $319 \pm$ $5 \mathrm{~K}$ ), indicating pronounced structural changes around this temperature. ${ }^{40}$

The good agreement between the experimental results shown in Figure 6A are in contrast to the relatively broad range of $\mathrm{O}-\mathrm{O}$ pair distribution functions obtained from molecular dynamics simulations using different effective water models (Figure 7). The recent diffraction measurements listed in Table 2, e.g., all give $2.44<g_{1}<2.62$, whereas TIP4P $/ 2005^{63}$ gives $g_{1} \sim 3.2$, and $\mathrm{mW}$ water ${ }^{65}$ gives a $g_{1} \sim 2.05$. Density functional theory and ab initio atomistic simulations also still struggle to reproduce the measurements within the experimental uncertainties (see Figure 7 and, e.g., the supporting information of ref 66).

2.6.3. Structure of Amorphous States of Water. An understanding of the numerous anomalies of liquid water is closely linked to an understanding of the phase diagram of the metastable noncrystalline states of ice. This phase diagram is constructed by excluding all crystalline phases and by showing the thermodynamically most stable noncrystalline form of water. Knowledge about this phase diagram is incomplete. Most notably there is a blank area, often called the "no-man's land", in which noncrystalline water crystallizes so rapidly that it is not possible so far to study it in experiments. Most interestingly, the lowtemperature part in this diagram seems to be a mirror image of the high-temperature part, in which liquid and supercooled water are the most stable noncrystalline forms (cf. Figure 4 in ref 69). However, the low-temperature part of this phase diagram is more complicated because the physical properties do not change continuously with increasing pressure, as is the case for the ambient temperature liquid. Instead, there are jump-like changes of properties such as density or isothermal compressibility, which 


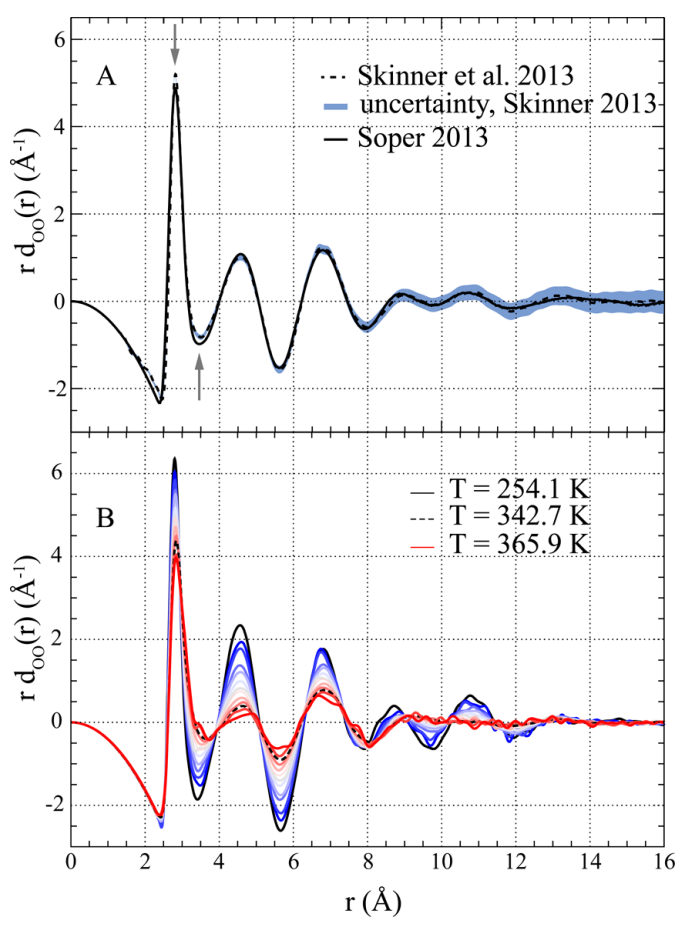

Figure 6. $\mathrm{O}-\mathrm{O}$ pair distribution function patterns $\left(r d_{\mathrm{OO}}(r)=\right.$ $\left.4 \pi \rho r^{2}\left[g_{\mathrm{OO}}(r)-1\right]\right)$, which emphasize the high- $r$ structure compared to the $g_{\mathrm{OO}}(r)$ function. (A) Room temperature patterns from Soper ${ }^{27}$ (solid black) and Skinner et al. ${ }^{11}$ (black dashed). The light blue area is the error range from ref 11 , and the arrows indicate the two $r$-regions where the two measurements slightly disagree. (B) $r d_{\mathrm{OO}}(r)$ patterns as a function of temperature from ref 40 . The black dashed line is at $342.7 \mathrm{~K}$ (the hottest containerless measurement), and the solid black line is at $254.2 \mathrm{~K}$ (the minimum temperature measured). The other colored lines correspond to a successive change of temperature, color coded from blue to red, at 263.2, 268.2, 277.1, 284.5, 295.2, 307.0, 312.0, 323.7, $334.2,354.9$, and $365.9 \mathrm{~K}$.

necessitate distinction among three distinct structural states of amorphous water. ${ }^{13}$ They are called low- (LDA), high- (HDA), and very- high- density amorphous ice (VHDA). ${ }^{70} \mathrm{LDA}$ itself can be prepared in different ways: by vapor deposition, ${ }^{71}$ by hyperquenching, ${ }^{72}$ and by transformation from HDA. ${ }^{13}$ HDA and VHDA can be prepared by applying high pressure of up to $1.6 \mathrm{GPa}$ at low temperatures $(77-160 \mathrm{~K}){ }^{13,73}$ As also indicated by their names, the amorphous states differ dramatically in density. Whereas LDA has a density lower than that of liquid water, HDA and VHDA both have a density that is higher compared to water-even at ambient pressure, where these forms of amorphous ice can be prepared metastably at low temperature. ${ }^{74} \mathrm{X}$-ray and neutron diffraction experiments play a key role in the characterization of the amorphous states of ice. The caveat in interpreting data deduced from static scattering

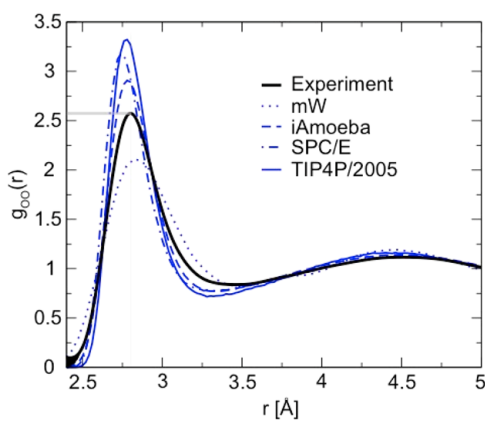

Figure 7. Comparison of $g_{\mathrm{OO}}(r)$ from measurements (thick black line ${ }^{11}$ ), and MD models (light blue lines). TIP4P $/ 2005^{63}$ (unbroken line) is the sharpest structure, followed by SPC $/ \mathrm{E}^{67}$ (dashed line) and iAmoeba, ${ }^{68}$ which is also over structured, while $\mathrm{mW}^{65}$ water (dotted line) is less structured than the measured $g_{\mathrm{OO}}(r)$.

methods is that it is hard to discriminate between an amorphous, but nanocrystalline material (unrelated to liquids) ${ }^{75,76}$ and an amorphous, glassy material (continuously connected to the liquid via the glass transition). Both cases do not show long-range correlation in their powder patterns. Using a variety of different dynamic scattering methods, also the glassy nature of amorphous ice has been investigated as described below. For a detailed discussion on the connection between LDA and HDA with deeply supercooled liquid water, we refer the reader to the recent review by Amann-Winkel et al. ${ }^{69}$

Here an overview on various measurements on the static structures and their temperature dependence is given. Early Xray and neutron wide-angle diffraction experiments on vapor deposited water (ASW), ${ }^{77,78}$ hyperquenched water (HGW), ${ }^{79,80}$ and $\mathrm{HDA}^{81-83}$ studied the structure of these different amorphous ices. Figure 8 shows the radial distribution functions of different amorphous ices as measured at the spallation source ISIS at the SANDALS spectrometer. ${ }^{13,70,84}$ The radial distribution functions were obtained using EPSR refinement as described by Bowron et al. ${ }^{70}$ LDA-II, eHDA, and VHDA are clearly distinct structural states. A first neighbor coordination number analysis of $g_{\mathrm{OO}}(r)$ shows ${ }^{70,84}$ LDA-II is 4 -fold coordinated, while the coordination number rises to $\approx 5$ in HDA and to $\approx 6$ in VHDA. There are one or two oxygen atoms occupying an "interstitial" nonbonded position within the first and second hydration shells in HDA and VHDA, respectively. ${ }^{70}$ The structures of UHDA and eHDA are very similar, as can be seen directly by comparing their $g_{\mathrm{OO}}(r)$ values (Figure 8). However, the two states show huge differences in their thermal behavior. Whereas uHDA shows relaxation processes preceding the uHDA $\rightarrow$ LDA transition, ${ }^{85,86}$ eHDA is a relaxed state transforming directly to LDA. ${ }^{87,88}$ This indicates that it is eHDA and not uHDA that serves as proxy for the liquid counterpart HDL (high-density liquid). ${ }^{69}$

Table 2. Average O-O Coordination Number $n_{\mathrm{OO}}(r)$ and First Peak Height $g_{1}$ As Determined by Three Different Studies ${ }^{a}$

\begin{tabular}{llccc}
\multicolumn{1}{c}{ study and method } & $n_{\mathrm{OO}}(3.25 \AA)$ & $n_{\mathrm{OO}}(3.3 \AA)$ & $n_{\mathrm{OO}}(3.36 \AA)$ & $g_{1}$ \\
Soper $^{27}(\mathrm{~N}+\mathrm{X}+\mathrm{EPSR})$ & & & $4.67 \pm 0.01$ & $2.49 \pm 0.05$ \\
Skinner et al. ${ }^{11}(\mathrm{~N}+\mathrm{X})$ & $4.14 \pm 0.10$ & $4.32 \pm 0.10$ & $4.54 \pm 0.10$ & $2.57 \pm 0.05$ \\
Zeidler et al. ${ }^{36}(\mathrm{~N})$ & $4.2 \pm 0.1$ & & & 2.49
\end{tabular}

${ }^{a}$ The coordination number $n_{\mathrm{OO}}(r)$ has been determined for different upper cutoff values $r$ which are indicated in parentheses. $\mathrm{N}$ denotes neutron diffraction, $\mathrm{X}$ denotes X-ray diffraction. The Zeidler et al. ${ }^{36} g_{\mathrm{OO}}(r)$ values are taken from their $\mathrm{H}-\mathrm{D}$ substitution neutron diffraction data. 

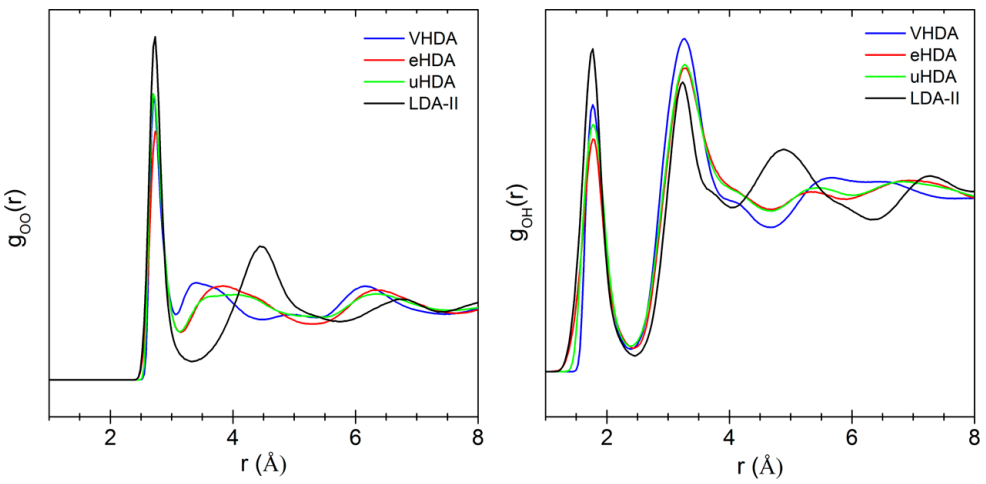

Figure 8. Comparison of $\mathrm{O}-\mathrm{O}$ and $\mathrm{O}-\mathrm{H}$ pair distribution functions of different amorphous ices: VHDA (blue), eHDA (red), uHDA (green), and LDA-II (black). Data have been obtained from neutron scattering measurements using EPSR structure refinement. ${ }^{13,70,84}$

In this context, ASW, HGW, LDA-I, and LDA-II ${ }^{84}$ represent substates of LDA, and UHDA and eHDA ${ }^{87}$ represent substates of HDA. These substates are prepared using differing pathways in the $p-T$ plane. While an infinite number of substates is possible, ${ }^{89}$ there are only three amorphous ices to which all substates relax. The key questions related to such substates are (i) to find the state of lowest potential energy in each megabasin (compare Figure 8 in ref 13) and (ii) to investigate whether they are homogeneous materials or not. The distinction can be made, e.g., on the basis of calorimetric ${ }^{90}$ or neutron diffraction measurements ${ }^{91}$ which show that LDA-II is more stable against crystallization than LDA-I or HGW (hyperquenched glassy water). Similarly, such measurements show eHDA to be more stable than uHDA against polyamorphic transition at ambient pressure by $\approx 25 \mathrm{~K}^{87,88}$ and by $\approx 10 \mathrm{~K}$ at elevated pressure. ${ }^{92,93}$ The lower thermal stability might be explainable by the presence of nanometer-scaled ice $\mathrm{I}_{\mathrm{h}}$ domains within the amorphous matrix in UHDA and LDA-I, whereas these domains are removed largely or even entirely in eHDA and LDA-II. The heterogeneous nature of UHDA on the nanometer scale was shown on the basis of small-angle neutron scattering by Koza et al. ${ }^{94,95}$ The heterogeneous character becomes visible by a pronounced small-Q signal in the SANS data. However, based on the interpretation by Seidl et al. ${ }^{92}$ eHDA should be homogeneous on the nanometer scale. The question of whether or not eHDA has a homogeneous character has not been tackled so far by smallangle scattering. By contrast, LDA-I and VHDA have been shown to be of homogeneous nature based on the SANS measurements. $^{94-96}$

Now, let us turn to the question of whether or not the two amorphous states HDA and LDA are connected to two distinct supercooled liquid phases. Since the discovery of HDA and LDA by Mishima et al. in $1985,{ }^{73}$ this question has been controversially discussed. Here we briefly discuss how diffraction methods might help to solve this key question, which is addressed in detail also in other review articles. ${ }^{69,97}$ In brief, experiments favoring the one interpretation can be found as readily as experiments favoring the other interpretation. Inelastic $\mathrm{X}$-ray scattering measurements indicate sharp crystal-like phonons in the amorphous ices, ${ }^{98}$ hence favoring the interpretation of amorphous ice being of nanocrystalline nature. ${ }^{75,76}$ Calorimetric measurements instead indicate glassy nature of LDA and a glass transition at $136 \mathrm{~K}^{99}$ and ambient pressure. Recently also the glassy nature and the glass transition in eHDA were observed at $116 \mathrm{~K}$ at ambient pressure ${ }^{4}$ and at 140 $\mathrm{K}$ at an elevated pressure of $1 \mathrm{GPa}^{100}$ using calorimetric measurements as well as dielectric spectroscopy. Measurements about the pressure dependence of HDA's glass transition are summarized in ref 101. Another method to detect a signature of the glass transition is quasi-elastic neutron scattering. Mode coupling theory predicts fast precursor processes prior to the glass transition temperature. These processes were found in molecular glass formers as well as in the hydrogen bond network glycerol. ${ }^{102}$ Quasi-elastic neutron scattering measurements on LDA-I and uHDA previously showed the absence of such fast precursor processes. ${ }^{103}$ Recently these experiments were repeated using LDA-II and eHDA. ${ }^{91}$ The Debye-Waller factor shows a very weak sub- $T_{\mathrm{g}}$ anomaly in some of the samples, which might be the signature of fast precursor dynamics and hence a signature for a glass transition. In any case, the question will certainly be at the origin of lively debates also in the future.

\section{WATER DYNAMICS FROM NEUTRON SCATTERING}

\subsection{Diffusive Dynamics}

The hydrogen bond (HB) adds a supramolecular length scale to the microscopic network structure of water leading to intriguing anomalies, with respect to simple liquids, not only in the thermodynamic and structural properties, but also in the dynamics.

In particular, the presence of $\mathrm{HB}$ and the related local tetrahedral structure directly affect the structural relaxation of the liquid, i.e. the molecule diffusivity, and the reorientational time scale at the molecular level.

The study of water diffusion at the microscopic scale thus represents a valuable piece of information for the understanding of fundamental molecular transport properties, such as water mass diffusion, ${ }^{2,14,104-110}$ hydrogen network relaxation, ${ }^{18,111}$ proton spin relaxation, ${ }^{112,113}$ and proton tunneling ${ }^{114-116}$ and can shed light on the peculiarity of the reorientational process of water molecules in a hydrogen bond network. ${ }^{117-122}$

While in the macroscopic hydrodynamic limit water diffusion is evaluated by tracer methods, and at the mesoscopic scale by field gradient NMR methods or dielectric measurements, evaluations at the molecular scale are possible either by model dependent NMR relaxation or by quasi-elastic incoherent neutron scattering (QENS). ${ }^{123}$ The latter directly measures the time dependence of the autocorrelation function of motions of hydrogen atoms exploiting the extreme sensitivity of neutrons to hydrogen, mainly owing to the largely incoherent scattering cross section of this element compared to that of other elements $\left(\sigma_{\mathrm{H}} \sim\right.$ 81 barn, other elements, $\sim 1.2$ barn). In particular, cold neutrons 

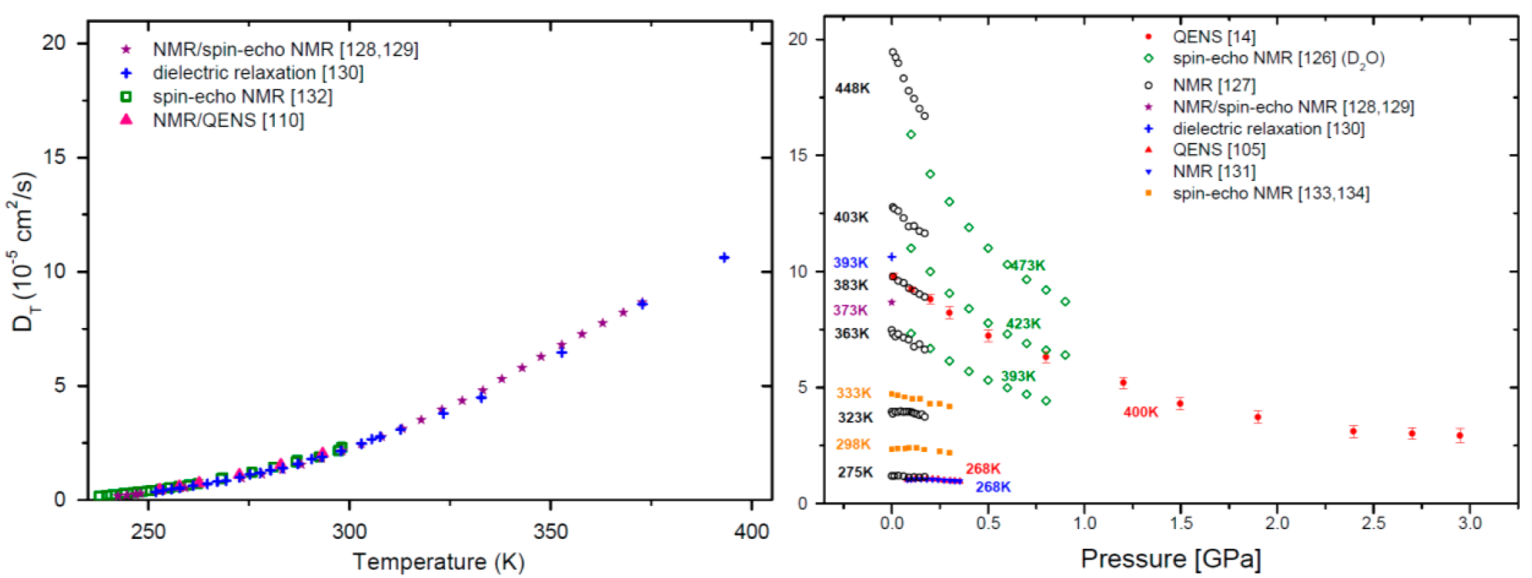

Figure 9. Translational diffusion coefficient for liquid water as a function of temperature (left panel) and of pressure (right panel), as derived from different techniques. ${ }^{14,105,110,126-134}$
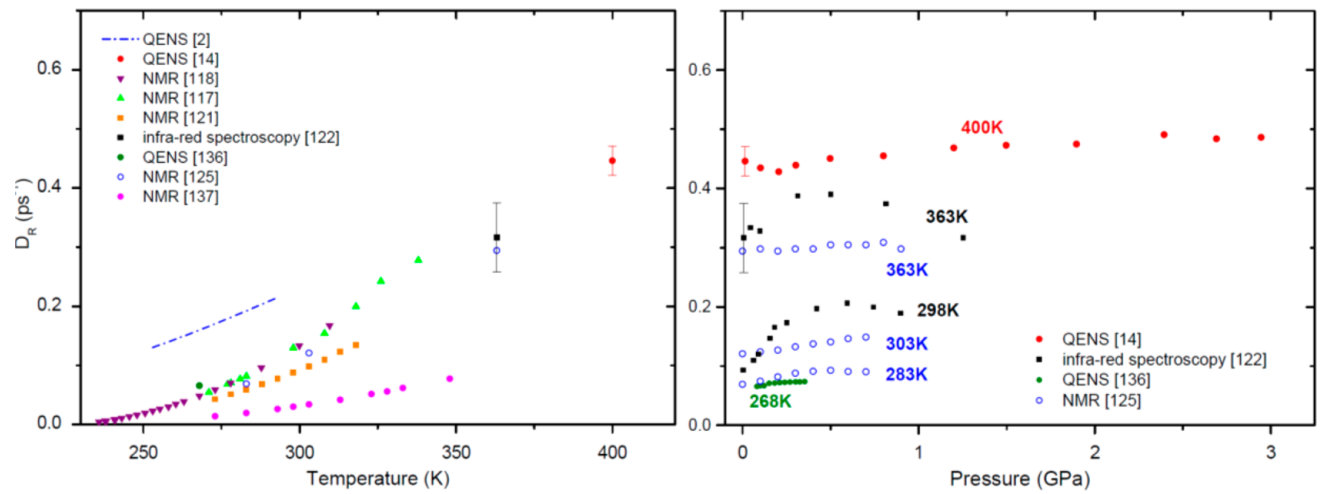

Figure 10. Rotational diffusion coefficient for liquid water as a function of temperature (left panel) and of pressure (right panel), as derived from different techniques. ${ }^{214,117,118,121,122,125,135,136}$

with wavelengths of a few angstroms and energies of several millielectronvolts allow investigations of molecular motions on a nano- to picosecond time scale. This powerful method has been used in extended regions of the domain of existence of the liquid state, including metastable conditions, in both supercooled ${ }^{2}$ and supercritical water ${ }^{106}$ (see Figures 9 and 10). Recent measurements employing a new high pressure device specially conceived for QENS studies ${ }^{14,124}$ have considerably extended in pressure (up to the multiple gigapascal range) our knowledge of the rotational and translational diffusion in liquid water, previously established in the kilobar range by high pressure NMR experiments $^{125}$ (Figures 9 and 10).

A big asset of the QENS technique is that the energy spectrum of the autocorrelation function $S_{\text {self }}(Q E)$ is measured as a function of the wave vector $Q$. This allows distinguishing more easily, compared to other techniques giving access to translational and rotational relaxation phenomena in the system, the specific nature of the observed motion by analyzing its $Q$ dependence. ${ }^{14}$ As an example, diffusion-like motions exhibit strong dependence of the decay rate $\Gamma$ (or relaxation time $\tau$ ) on $Q$ while for local relaxation processes the characteristic $\Gamma$ (or time scale $\tau$ ) is independent of $Q$ at least at large $Q$.

Thus QENS data are potentially rich in information, but there is currently no consensus on their interpretation in HB systems. Data analysis of QENS data on water has been done essentially within three different models, which take into account intramolecular motions in different ways:

1. The first model is expressed in terms of a decomposition of the molecular center of mass motion and rotational motions, with the last being reduced to large amplitude displacements of hydrogen atoms associated with hydrogen bond dynamics. ${ }^{2}$

Within this assumption of a roto-translational decoupling and of a multiexponential time decay of the density-density correlation function, the (self) incoherent dynamic structure factor can be cast into the following form:

$$
\begin{aligned}
& S_{\mathrm{S}}(Q, E)=\mathrm{e}^{-\left\langle u^{2}\right\rangle Q^{2} / 3}\left[\frac{j_{0}^{2}(Q a)}{\pi} \frac{\Gamma_{\mathrm{T}}}{E^{2}+\Gamma_{\mathrm{T}}^{2}}\right. \\
& \left.+\frac{1}{\pi} \sum_{l=1}^{\infty}(2 l+1) j_{l}^{2}(Q a) \frac{l(l+1) D_{\mathrm{R}}+\Gamma_{\mathrm{T}}}{E^{2}+\left[l(l+1) D_{\mathrm{R}}+\Gamma_{\mathrm{T}}\right]^{2}}\right]
\end{aligned}
$$

where the exponential term is the analogue of the Debye-Waller factor of solids, $\left\langle u^{2}\right\rangle$ represents the single-proton vibrational amplitude, $j_{l}(x)$ is the spherical Bessel function of $l$-order, $a$ is the radius of rotation (for water fixed to the $\mathrm{O}-\mathrm{H}$ distance), $\Gamma_{\mathrm{T}}$ is the translational half-width at half-maximum, and $D_{\mathrm{R}}$ is the rotational diffusion coefficient.

The Q-dependent translational parameter $\Gamma_{\mathrm{T}}(Q)$ is usually observed to be smaller, at high momentum transfer, $Q$ than 
expected from a continuous translational diffusion $\left(D_{\mathrm{T}} Q^{2}\right),{ }^{86}$ in agreement to what is predicted by a continuous random walk model. ${ }^{110}$ In the low- $Q$ range explored by QENS experiments, the $Q$-dependence of $\Gamma_{\mathrm{T}}(Q)$ is often described as $\Gamma_{\mathrm{T}}=D_{\mathrm{T}} Q^{2} /(1$ $\left.+d^{2} Q^{2} / 6\right)$, with $d$ representing an apparent jump length. Following the continuous time random walk (CTRW) model, the jumps responsible for the noncontinuous $Q$ dependence of $\Gamma_{\mathrm{T}}(Q)$ can be identified as transitions between the dynamical basins model. ${ }^{110}$

2. Assumption of a distribution of relaxation times that generates a nonexponential decay of the hydrogen correlation function is the second model. ${ }^{137}$ This model is based on the application of the mode coupling theory (MCT) to the analysis of the time decay of the density-density correlation function of liquid water. The MCT theory has been successfully applied to describe the dynamics in polymer melts ${ }^{138}$ and other hydrogenbonded systems. This model, even if, in order to be applied to QENS data analysis, it is formally more complex than it implies to perform a Fourier transform of the experimental dynamic structure factor to extract the time dependent density-density correlation function, has the advantage of highlighting the normal (Arrhenius) temperature dependence of hydrogen bond dynamics down to extremely low temperatures. The $\beta$ relaxation in water is in fact identified with the HB dynamics, which has a very short time even at the homogeneous nucleation temperature and drives the glass transition of the liquid at much lower temperature. This is in contrast to what happens with polymer gels, where the $\beta$ processes, related to backbone movement, block the fast molecular motions. Instead, the anomalous (nonArrhenius) temperature dependence of the $\alpha$ relaxation dominates the transport properties, namely the shear viscosity, as in a polymer melt, at higher temperatures. It yields crystallization because, in a percolation process, hydrogen bonds are formed with the same tetrahedral symmetry that characterizes hexagonal ice.

3. The third approach is based on extensive coarse grain simulations that use an effective potential (SPCE). This method, recently applied on high-quality QENS data, ${ }^{110}$ leads to the identification of two highly entangled motional components, corresponding to two distinct types of structural dynamics: picosecond local (L) structural fluctuations within dynamical basins and slower interbasin jumps $(\mathrm{J})$. However, it must be noticed that these two times are not directly associated with precise diffusion mechanisms. ${ }^{91}$ This model essentially does not take into account water rotations and is based on a rigid molecule approximation that thus neglects any independent motion of the hydrogen atoms. This constitutes mainly a limitation at high temperatures, when water rotations have the same time scale (picoseconds) as the mass diffusion motions and vibrations ${ }^{110}$ but provides a more accurate description of the low temperature regime with respect to the roto-translational model.

The continuous roto-translational model, even if it is known to be fundamentally inadequate as it assumes a complete decoupling between diffusional and rotational motions, has the advantage of providing a straightforward analytical model to fit over the data, allowing extraction of translational and rotational time scales. ${ }^{2,14,104,105,107,139}$ However, it should be remarked that, as the neutron couples with individual protons, in QENS measurements context the word "rotation" refers to individual motions of hydrogen, which would coincide with molecular rotations only if the molecule was rigid. Such an assumption is currently made, namely in molecular dynamics simulations.
However QENS measures as well large displacements of hydrogen atoms that break a single hydrogen bond.

The comparison of microscopic diffusion coefficients derived by QENS data with the "mesoscopic" quantities derived by other techniques is not always straightforward (see Figures 9 and 10). NMR and QENS results almost coincide, although coupling in a different way with molecules, while dielectric and field gradient NMR methods often provide disparate results, both in temperature and in pressure.

Of note is that Laage and co-workers ${ }^{119,120}$ have recently shown that liquid water quasi-elastic neutron scattering spectra in the continuous roto-translational model generally underestimate the water reorientational time. In order to compare results estimated via other techniques such as NMR and ultrafast infrared spectroscopies with QENS results, an extended jump model for water rotation ${ }^{119,120}$ has been applied (Figure 9). This model predicts a ratio between the hydrogen self-motion around the molecular center of mass, measured by QENS, and the reorientational time of the molecular dipole, measured by NMR or dielectric technique, closer to 2 , rather than 3 , as predicted by the continuous model. ${ }^{2}$

The availability of new high-quality QENS data on water in an extended temperature and pressure domain is an important source of information to distinguish between different valuable phenomenological approaches and in order to disentangle density and temperature effects.

In particular, applying considerably high pressures to distort the local structure without destroying the $\mathrm{HB}^{140}$ is a powerful tool to test the dependence of water rotation time from first neighbor local geometry and/or from the surviving time of the H-bonds.

QENS and time-resolved infrared measurements on hot dense water $^{14,122}$ have shown that the translational and rotational diffusion coefficients are unexpectedly uncorrelated under pressure. In particular, rotational diffusion is almost unaffected by pressure (Figure 10), as the first coordination shell, the number of hydrogen bonds, and their strength do not vary in the compressed liquid measurements. ${ }^{141,142}$ Conversely, the translational diffusion is slowed down under pressure, as a consequence of the free volume reduction (Figure 9).

A comparison between self-diffusion at the molecular scale and transport properties is interesting because it establishes a precise relation between hydrodynamics and molecular motions. Very often, the Stokes-Einstein relation describes remarkably well such a relation between self-diffusion and viscosity through a characteristic length. However, QENS measurements in undercooled $^{143}$ and high-density water ${ }^{14}$ show that this relation breaks down in water, where the local structure and the available free volume for diffusion are determined by the HB network rearrangement under variation of the thermodynamic parameters.

\subsection{Vibrational Dynamics}

3.2.1. General Features. One of the basic functions used to characterize atomic dynamics is the vibrational density of states (VDOS). In general, it can be extracted from an analysis of the isotropic incoherent dynamical structure factor $S_{S}(Q E)$, which in hydrogen-rich samples is proportional to the double differential cross section, measured by neutron scattering experiments. ${ }^{144}$ Here, $E$ is the exchanged energy and $\hbar Q$ is the modulus of the exchanged momentum. In more detail, the scattering process is very sensitive to the space-dynamical characteristics of the system, as the typical energies of thermal neutrons are similar to 
the vibrational mode energies $(1-500 \mathrm{meV})$ and the neutron wavelength is comparable to the interatomic distances in condensed materials. The inelastic incoherent neutron scattering (IINS) spectrum is directly proportional to the VDOS, where the contribution from each vibrational mode is weighted by the relevant atomic mean-square displacements and by the neutron scattering cross sections of the constituent atoms. Hence, IINS measures all vibrational modes simultaneously and the spectrum provides direct information about intermolecular and intramolecular interactions. As mentioned before, in the case of water the signal comes mostly from the incoherent cross section of hydrogen atoms, so the VDOS can be written as $G(E)=\sum_{j} \frac{1}{3 M}\left|\boldsymbol{e}_{j}\right|^{2} \delta\left(E-\hbar \omega_{j}\right)$, where $\boldsymbol{e}_{j}$ is the polarization vector of normal modes at the hydrogen sites and $M$ is the hydrogen atomic mass. In the one-phonon approximation ${ }^{144}$ the VDOS is related to the dynamical structure factor by the relationship

$$
\begin{aligned}
S_{\mathrm{S}}(Q, E)= & \frac{\sigma_{\mathrm{H}}^{\mathrm{inc}}}{4 \pi} \mathrm{e}^{-2 W(Q)} \frac{\hbar^{2} Q^{2} G(E)}{2 M E}[n(E) \\
& \delta(E+\hbar \omega)+(n(E)+1) \delta(E-\hbar \omega)]
\end{aligned}
$$

where $W(Q)$ is the so-called Debye-Waller factor for the hydrogen atoms and $n(E)$ is the Bose-Einstein factor.

3.2.2. Intermolecular Vibrations. In the energy range up to $150 \mathrm{meV}$, the contribution to the VDOS mainly comes from intermolecular motions. The bulk water VDOS is characterized by the presence of three main bands. ${ }^{145,146}$ At $6 \mathrm{meV}$ a rather broad peak represents the collective modes of hydrogen-bond bending (HBB), perpendicular to the line formed by the hydrogen bond $\mathrm{O}-\mathrm{H} \cdots \mathrm{O} .{ }^{147}$ This mode is reminiscent of the transverse acoustic mode TA1 sustained by the $\mathrm{O}-\mathrm{O}-\mathrm{O}$ bending mode in crystalline hexagonal ice (Ih). ${ }^{148}$ Then, the region between 20 and $35 \mathrm{meV}$ is characterized by a very weak band, which is attributed to hydrogen-bond stretching (HBS) modes parallel to the line formed by the hydrogen bond $\mathrm{O}-\mathrm{H} \cdots$ O. ${ }^{149}$ This feature appears as a broad shoulder beside the HBB band, while it is much more visible in Raman spectra. ${ }^{150} \mathrm{HBB}$ and HBS bands correspond to the vibrational, i.e. phonon-like, translational dynamics of water. At higher energies the librational motion of water molecules gives rise to a quite intense band in the range $50-130 \mathrm{meV}$ (LIB). ${ }^{151,152}$

The behavior of these HBB, HBS, and LIB bands has been also studied in supercooled water, ${ }^{146}$ confined water, ${ }^{153}$ and water at the interface with proteins ${ }^{16,154}$ and DNA. ${ }^{155}$

Particularly the HBB band is quite sensitive to the local environment, so it is significantly suppressed when water is confined, due to the reduction of translational degrees of freedom compared to the bulk. ${ }^{153}$ At low temperature water at the interface with proteins ${ }^{156}$ or with moieties with a more or less predominant hydrophobic character, such as $\mathrm{N}$-acetyl-leucinemethylamide (NALMA) and $\mathrm{N}$-acetyl-glycine-methylamide (NAGMA), respectively, ${ }^{17}$ shows a HBB band whose shape is very similar to amorphous ice and quite different from the crystalline phase. In general, the HBB peak seems to be quite sensitive to the local topology of water molecules. Hydrostatic pressure has been shown to give rise to an appreciable blue shift of the position of the HBB band of bulk water, ${ }^{157}$ possibly due to the stiffening of the environment felt by water molecules. Interestingly, as shown in Figure 11, the VDOS of protein hydration water is much more similar to that at the interface with NALMA than NAGMA, despite the fact that polar amino acids

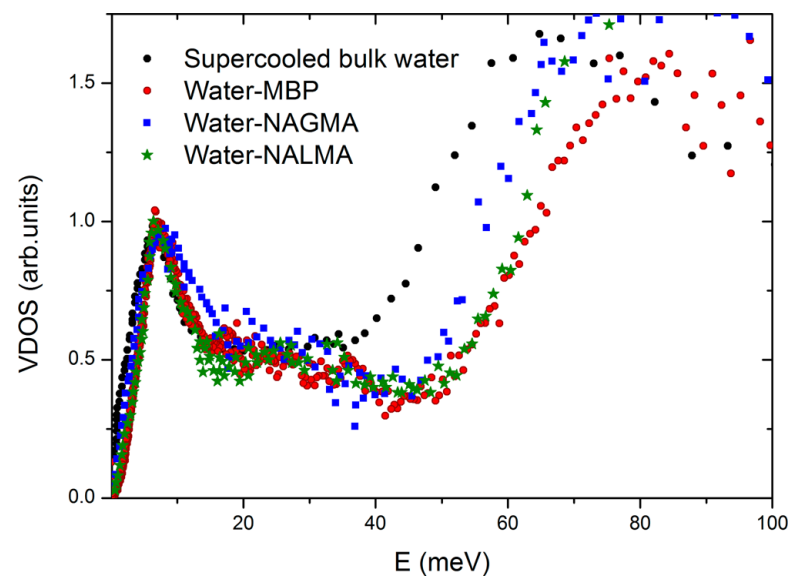

Figure 11. Vibrational density of states of supercooled water measured on the IN6 spectrometer (Institut Laue-Langevin, Grenoble) ${ }^{145}$ and water at the interface with maltose binding protein (MBP), ${ }^{16,156}$ NALMA, and NAGMA molecules, ${ }^{17}$ measured on the IN5 spectrometer (Institut Laue-Langevin, Grenoble). The VDOS curves have been normalized to the first inelastic peak at $6 \mathrm{meV}$.

are usually found at the protein surface. Also, Figure 11 shows that the left edge of the LIB band in the spectra of lowtemperature water at the interface with biomolecules ${ }^{16,156}$ is redshifted compared to supercooled water, a behavior much closer to HDA ice than ice Ih. ${ }^{158}$ In particular, the VDOS of amorphous forms of ice also shows three smooth bands that can be connected to the HBB, HBS, and LIB features, ${ }^{159}$ at variance with the case of Ih crystalline ice, where much more sharp and structured inelastic bands appear in the translational region at 7.1, 13.3, 19, 28.4, and $37.9 \mathrm{meV}$, with librational modes well confined between 67 and $121 \mathrm{meV}$.

3.2.3. The Boson Peak. A quite debated point is the possible existence of a Boson peak in the low-energy region of inelastic neutron scattering spectra of amorphous ice, supercooled water, and interfacial water. The Boson peak is an excess of modes in the range from 2 to $10 \mathrm{meV}$ over the low-energy flat Debye level, following the thermal occupation of a Bose system, i.e., phonons. It can be observed in the reduced VDOS $g(E) / E^{2}$ representation, or quite equivalently via the dynamical structure factor, of glasses and amorphous solids. ${ }^{160}$ In the case of amorphous water, the measurement of a possible Boson peak is made difficult, mainly because the low-energy range is dominated by the HBB feature. Even though experimental evidence has been provided in the past for the presence of a Boson peak in HDA ice, ${ }^{161}$ more recent neutron time-of-flight and backscattering spectroscopy results seem to rule out this possibility. ${ }^{162}$ On the other hand, a Boson peak has been found in experiments on and simulations of protein hydration water, ${ }^{163,164}$ simulations of supercooled water, ${ }^{165}$ and in supercooled confined water. ${ }^{143}$ Actually, in most of these investigations the Boson peak appears as a broad bump centered at $4-5 \mathrm{meV}$ in the dynamical structure function. Since in the VDOS representation this feature would appear somehow blue-shifted to about 6-7 $\mathrm{meV}$, it can be argued that the Boson peak of interfacial and supercooled water can be likely identified with the HBB band. The traditional distinction between the two, the Boson peak and the HBB band, is probably fictitious and results mainly from the fact that they are usually observed in the dynamical structure factor and in the VDOS representations, respectively. Quite recently an interesting 
picture has been suggested where the Boson peak would be the glassy counterpart of the van Hove singularity of the corresponding crystal, with the density of the system, and not the disorder, playing a critical role. ${ }^{166}$ This would imply that the Boson peak, i.e. the HBB band, is nothing but the glassy counterpart of the TA1 mode in the zone-edge range of phonon dispersion curves of ice. ${ }^{148}$

3.2.4. Intramolecular Vibrations. In the energy range above $150 \mathrm{meV}$, the contribution to the VDOS comes from intramolecular motions. The fundamental $\mathrm{O}-\mathrm{H}$ bending and stretching modes of bulk water at $40{ }^{\circ} \mathrm{C}$ are well visible at about 207 and $435 \mathrm{meV} .{ }^{167}$ This latter band shifts to a lower energy value of $428 \mathrm{meV}$ in supercooled water, due to the formation of a more stable hydrogen bond network between neighboring molecules which makes a softer intramolecular attractive bond. ${ }^{167}$ In the spectra also two bumps at 280 and $520 \mathrm{meV}$ appear, which are the combination bands between the bending and the librational modes and between the stretching and the librational modes, respectively. ${ }^{151}$ It is worth noting that both the position and the intensity of the bending and the stretching vibrational modes are quite sensitive to structural changes, confinement, or interaction with polar groups. In fact, highpressure crystalline and amorphous phases of ice show significant differences in the intramolecular vibration range compared to Ih ice. $^{158,159}$ The blue shift of the $\mathrm{O}-\mathrm{H}$ stretching mode of water interacting with biomolecule ${ }^{168}$ or oxide ${ }^{169}$ surface, or confined within carbon nanotubes, ${ }^{170}$ is consistent with the weakening of the hydrogen-bonded water network in these systems.

\subsection{Collective Dynamics}

3.3.1. General Features. The collective dynamics in water corresponds to high-frequency modes with wavelengths close to interatomic separations. Since these are the eigenstates of a topologically disordered system, these modes are phonon-like in a sense that they are not entirely harmonic vibrations as in crystals. These short wavelength collective excitations manifest themselves as inelastic peaks in the measured coherent signal. Because of the finite experimental energy resolution, these excitations appear most of time as shoulders on each side of the main central elastic peak. In addition, since their damping increases with the exchanged vector, collective modes are generally much more visible for low- $Q$ values of the coherent structure factor $S(Q, E)$. By reporting the excitation energies of these modes as a function of $Q$ the so-called dispersion curves measured by inelastic X-ray scattering (IXS) and inelastic neutron scattering (INS) are represented in Figure 12, respectively, for bulk water and water at the interface with biomolecules. The details of the two branches appearing in the picture are discussed in sections 3.3 .2 and 3.3.3. It has to be acknowledged that, despite considerable effort, a commonly accepted scenario in this field is still missing. Actually, the description of the high-frequency collective dynamics requires the deep understanding of the interplay between collective properties such as density, charge, and concentration fluctuations, and other phenomena such as particle diffusion, particle rotations, and the degrees of freedom associated with the internal structure of the particle itself, that are typical of the fluid state.

3.3.2. Fast Sound. In the low momentum-transfer range $(Q$ $<0.003 \AA^{-1}$ ) bulk water at ambient conditions exhibits a single longitudinal acoustic (LA) mode, propagating at a velocity equal to the adiabatic one $c_{S}{ }^{179}$ At higher $Q$ values, a positive sound dispersion, i.e. a large increase of the speed of sound up to more than $3000 \mathrm{~m} / \mathrm{s}$, was first observed by INS. ${ }^{171,172}$ In the following,

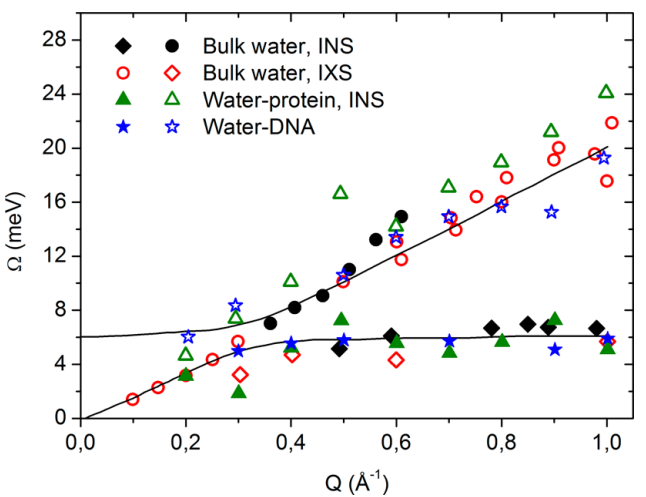

Figure 12. Dispersion curves of bulk water and water at the interface with biomolecules. The excitation energies of bulk water were measured with both INS (black closed rhombuses, ${ }^{171}$ black closed circles ${ }^{172}$ ) and IXS (red open rhombuses, ${ }^{173,174}$ red open circles ${ }^{175}$ ). The excitation energies of water at the interface with RNase protein ${ }^{176}$ (closed and open green triangles, for the low- and high-energy branches, respectively) and $\mathrm{DNA}^{177}$ (closed and open blue stars, for the lowand high-energy branches, respectively) were measured by INS. The continuous line represents the two-mode interaction model. ${ }^{178}$

the occurrence of this phenomenon, also called "fast sound", was measured in more detail by ultrahigh resolution IXS. ${ }^{173}$ Still using IXS, it was found that the velocity of sound ${ }^{174}$ is equivalent in liquid and solid (ice $\mathrm{Ih}$ ) water in the investigated $Q$ region from 0.4 to $1.4 \AA^{-1}$. The transition from normal to fast sound has been suggested to occur in the $Q$ range from 0.1 to $0.4 \AA^{-1}$, with the fast sound representing the propagation of a collective mode in a solid-like network of molecules occurring at frequencies larger than the reciprocal of the liquid relaxation time scale. ${ }^{175} \mathrm{In}$ more detail, for $Q \leq 0.2 \AA^{-1}$ the IXS spectra were explained in terms of a hydrodynamic formalism which includes a viscoelastic, $Q$-independent contribution to the memory function for the density fluctuations. ${ }^{180,181}$ This model has been applied to bulk water in a wide temperature range (273-473 K), using pressure from 0 to $1.5 \mathrm{kbar}$ to keep the density constant at about $1 \mathrm{~g} / \mathrm{cm}^{-3}$, and recently in an extended $P$-range $(1-30 \mathrm{kbar})$ at $450 \mathrm{~K}^{3}$ to vary density up to $1.5 \mathrm{~g} / \mathrm{cm}^{-3}$. In this low- $Q$ range, the derived values for the fast sound velocity and for the structural relaxation time were found comparable with those obtained using techniques sampling a lower-frequency range. On the other hand, the viscoelastic contribution to the memory function ${ }^{180}$ was found to be $Q$-dependent for $Q>0.2 \AA^{-1}$ at constant density and linearly dependent on density at constant temperature.

It is worth mentioning that the existence of short-lived coherent excitations with a speed of about $3500 \mathrm{~m} / \mathrm{s}$ has been proven also for water at the interface with proteins and DNA, ${ }^{176,177,182}$ in agreement with MD simulation results. ${ }^{164,183}$ A distinct feature of water at the interface with biomolecules is the rapid overdamping of the high-frequency mode, which supports the glass-like behavior of bound water. The collective vibrational features of biological water does not seem to change when the complexity of the investigated systems increases, as it arises from studies performed on bacteria ${ }^{184}$ and human cells. ${ }^{185}$

3.3.3. Low-Frequency Branch. Apart from the fast sound propagating mode, a second low-frequency and weakly dispersing mode already predicted by MD simulation ${ }^{186}$ can be seen in the terahertz $(\mathrm{THz})$ spectrum of water by both INS $^{171,172,178}$ and IXS ${ }^{175,187-189}$ experiments. The nature of this mode is quite debated. At first it has been supposed to have a 
transverse optic character, due to the similarity with the low-lying optic mode of ice Ih. ${ }^{175}$ Successive experimental and theoretical investigations pointed out the transverse acoustic character of this excitation. ${ }^{187,190}$ Particularly this character has been suggested to hold for water in different thermodynamic conditions, including the supercooled liquid phase, in the $Q \leq$ $Q_{\mathrm{MAX}} / 2 \approx 0.8-0.9 \AA^{-1}$ range, where $Q_{\mathrm{MAX}}$ is the position of the first sharp diffraction peak in the static structure factor $S(Q){ }^{188}$ On the contrary, above $Q_{\text {MAX }}$, this prevalent transverse acoustic nature cannot be proved anymore. A further characterization of the $\mathrm{THz}$ spectrum of water has been done quite recently by combining INS and IXS, to take advantage of both the narrow and sharp energy resolution of the former and the wide explored dynamic range explored by the latter. ${ }^{181,189}$ In this investigation it is shown that the low-frequency peak is strongly overdamped in the low- $Q$ regime, where the dominant frequency of this transverse mode turns out to be comparable with the inverse of the structural relaxation time. Conversely, in the high- $Q$ regime the excitation is clearly resolved in the spectrum as an inelastic peak.

It is worth mentioning that in an alternative picture the water collective dynamics has been described by a model, where the observed dispersion curves arise from the interaction between optic-like and solid-like fast acoustic branches, coupling for $Q$ values around $0.3-0.35 \AA^{-1}$, i.e. the wave vector region where the transition from normal to fast sound occurs. ${ }^{178}$ Both the relaxation and the two-mode interaction schemes suggest the interaction mechanisms of the density fluctuation mode with the system as a whole to be the key to explaining the sound propagation in water.

\section{CONCLUSION: GOING FURTHER INTO THE KNOWLEDGE OF THE STRUCTURE AND DYNAMICS OF WATER IN AMORPHOUS STATES AND UNDER EXTREME CONDITIONS OF TEMPERATURE AND PRESSURE}

With the advent of a new generation of accelerator based pulsed neutron sources like the European Spallation Source (ESS), opportunities to improve the knowledge of the structure and dynamics of water are expected due to the higher flux as compared to current neutron sources like ISIS and ILL. It could be possible to approach the interatomic potential of water because the potentials used in simulation are almost always validated by comparison with neutron scattering data. Similarly, the development of X-ray lasers such as the Linac Coherent Light Source (LCLS) in the United States and the SPring-8 Angstrom Compact free electron LAser (SACLA) in Japan together with upcoming other sources allows completely new measurements of water using the coherent properties and the ultrashort pulse length.

\subsection{Improvement for the Structure of Water}

The application of polarization analysis has so far been limited by the available intensities at reactor sources; for example, for $\mathrm{H}_{2} \mathrm{O} 1$ week of neutron beam time is required. The promise of full polarization analysis at ESS instruments will allow us to separate coherent and incoherent contributions, ${ }^{191,192}$ to remove the incoherent scattering of hydrogen and associated inelastic scattering for $\mathrm{H}_{2} \mathrm{O}$ in order to get the coherent structure factor with an increased accuracy. In the case of isotopic substitution one expects to reach partial structure factors with a higher precision.
Structural measurements under extreme conditions of temperature and pressure as well as in levitation become now possible due to the higher neutron flux.

Another interesting possibility is to do in situ and real-time measurements of structural changes, for example, at low temperature and in the supercooled state of water. Even if these measurements are already possible with the existing synchrotron facility as noted previously it is important to use the complementarity between X-ray and neutron techniques.

Recent work at LCLS ${ }^{12}$ has allowed single shot X-ray laser measurements of fast cooled water droplets down to temperatures into the deep supercooled regime at time scales faster than ice nucleation. The intensity of each X-ray pulse was high enough in a single shot for the detection of a diffraction pattern of micrometer sized water samples. This can be further developed to probe water at various extreme conditions where the stability of the sample is very short.

From the utilization of the coherent properties of the X-ray laser beam, potentially higher correlation functions can be derived going beyond pair correlations. In particular, angular correlations at a specific momentum transfer could shed insights into various symmetries of local configurations. ${ }^{193}$

\subsection{Improvement for the Dynamics of Water}

It will provide in the near future the possibility to probe smaller and smaller samples, and thus to reach more extreme conditions of temperature and pressure by using dedicated high-pressure devices and levitation techniques. The availability of high quality QENS data, directly probing proton dynamics in an extended pressure and temperature domain, will help to discriminate the most appropriate description for water molecular diffusion and will possibly contribute to shedding light on the still-debated question of whether water dynamical anomalies are linked to possible critical phenomena in the undercooled ${ }^{12,194,195}$ or dense regime. ${ }^{14,15,122}$ The possibilities of measuring proton quantum effects at low temperatures ${ }^{114,196}$ and to probe the link between the proton dynamics and the breaking of the hydrogen bond under different thermodynamic conditions ${ }^{3,14}$ definitively deserve to be further explored.

$\mathrm{X}$-ray photon correlation spectroscopy (XPCS) is the application of dynamic light scattering in the X-ray regime ${ }^{197}$ and utilizes the high coherence of the novel X-ray laser sources ${ }^{198}$ together with a short pulse structure allowing for the determination of dynamics. The key is to resolve the X-ray speckle pattern and follow how it develops in time. A speckle pattern is simply intensity fluctuations of scattered light, caused by interference between the wavefronts that originate from the scattering of a coherent beam on individual atoms. This will allow the determination of real water equilibrium dynamics on time scales ranging from tenths of femtoseconds to minutes and length scales from nearest-neighbor distances to several nanometers.

\section{AUTHOR INFORMATION}

\section{Corresponding Author}

*E-mail: marie-claire.bellissent-funel@cea.fr.

\section{Author Contributions}

The manuscript was written through contributions of all authors. All authors have given approval to the final version of the manuscript.

Notes

The authors declare no competing financial interest. 


\section{Biographies}

Katrin Amann-Winkel studied physics at the Technical University Darmstadt. She completed her Ph.D. under the supervision of Erwin Mayer and Thomas Loerting on amorphous ices in 2009 at the University of Innsbruck. She continued her studies on the glass transition of amorphous ices in Innsbruck with a Hertha Firnberg grant (FWF) until 2014 and then moved to Stockholm University, working now in the group of Anders Nilsson. Her work was awarded with several prizes, such as recently the "Fritz Kohlrausch Preis 2014" from the Austrian Physical Society.

Marie-Claire Bellissent-Funel received her degree in physics from the University of Paris and her Thesis of Doctor ès Sciences Physiques from the University of Grenoble (France) in 1977. She taught at the University of Grenoble before joining the Centre National de la Recherche Scientifique (CNRS). Presently, she holds the position of Director of Research Emeritus at the Laboratoire Léon Brillouin where she was in charge of the research group centered on biology and disordered systems. She has been elected a Fellow of the Institute of Physics (U.K.) and granted the title of Chartered Physicist. On August 2000, she chaired the Gordon Research Conference on Water and Aqueous Solutions. From the International Academy of Lutèce, she received in 2006 the "Grand Prix" of Sciences for her novel work on water in confined media. She was invited as a Scientific Advisor at Helmholtz Zentrum Berlin for two years in January 2008. Her current research interests are water related and use different experimental (neutron and X-ray scattering, biophysical techniques) and modeling approaches. They include the structural and dynamic properties of bulk water in various conditions of temperature and pressure, the properties of water in confinement, the role of water in the stability and function of biological macromolecules, and the study of thermal and pressure denatured states of proteins.

Livia Eleonora Bove obtained her Ph.D. degree in physics from the University of Perugia in Italy in 2001. Afterward, she joined the Institut Laue-Langevin in Grenoble, France, as a postdoc, first, and as staff scientist since 2003. She became a researcher at CNRS in 2005, and she joined the IMPMC laboratory in Paris where she started working on water and aqueous solutions under extreme conditions. She is associated with the Earth and Planetary Science Laboratory at the EPFL in Switzerland, as an invited scientist. She has been involved with neutron instrumentation and research for the past 18 years. Her main field of interest today deals with studies on water, aqueous solutions, and gas hydrates under extreme conditions of pressure and temperature, including polyamorphism phenomena, proton ordering, nuclear quantum effects, water dynamics, and new exotic properties of water and ices developed under extreme conditions.

Thomas Loerting was born in 1973, studied chemistry at the University of Innsbruck in Austria, and obtained his Ph.D. in theoretical chemistry under the supervision of Prof. Klaus R. Liedl in 2000. As a postdoc he changed from theory to experiments and worked with Prof. Erwin Mayer in Innsbruck and Nobel laureate Prof. Mario J. Molina at the Massachussetts Institute of Technology. After his Habilitation in Physical Chemistry in 2008 he became associate professor and speaker of the Research Platform "Material- and Nanosciences" at the University of Innsbruck and member of the Austrian Academy of Sciences ("Junge Kurie"). His group's research on cryochemistry, supercooled water, amorphous and crystalline ices and clathrate hydrates was honored with a dozen awards, including the Starting Grant of the European Research Council (ERC), the START-Preis of the Austrian federal ministry of research and sciences, the Bodenstein award of the German Bunsen society, and the Bessel award of the Alexander von Humboldt
Foundation. Recently, he was visiting scientist at TU Dortmund University, collaborating with Prof. Roland Böhmer.

Anders Nilsson received a Ph.D. in physics at Uppsala University, Sweden (1989), in the laboratory created by Kai Siegbahn. He is currently professor in chemical physics at Stockholm University and in photon science at the SLAC National Accelerator Laboratory and Stanford University. He received the Lindbomska Award at the Swedish Royal Academy of Science, the Royal Oscar Award at Uppsala University in 1994, the Shirley Award at Berkeley in 1998, and the Humboldt Award for senior scientist in 2010, and was awarded honorable doctor at Denmarks Technical University in 2015. His research interests include synchrotron radiation and X-ray laser spectroscopy and scattering, chemical bonding and reactions on surfaces, ultrafast science heterogeneous catalysis, electrocatalysis in fuel cells, photocatalysis for converting sunlight to fuels, structure of water, and aqueous solutions.

Alessandro Paciaroni received his master's degree in physics with first class honors in 1993 and his Ph.D. degree in physics in 1996 at the University of Perugia (Italy), by using neutron scattering techniques to study structural properties of magnetic materials. After two years of postdoctoral research, where he combined neutron scattering and molecular dynamics simulation to study the dynamical properties of protein hydration water, he moved to Grenoble (France) where he became researcher for the Italian National Research Council at the international neutron source Institut Laue-Langevin. In 2001 he returned to Perugia to join the Department of Physics where he is now professor. His research spans two major areas: studying the coupling between water and biomolecules and understanding the relationships of protein dynamics to structure, stability, and function.

Daniel Schlesinger studied physics at the Ludwig-Maximilians University (LMU) of Munich with studies abroad at Lomonosov Moscow State University and graduated from LMU with a diploma in physics in 2009. He then joined the group of Lars G. M. Pettersson and Anders Nilsson at Stockholm University for Ph.D. studies and obtained his Ph.D. in October 2015. His main interests include molecular simulations, liquid state theory, and, in particular, the local structure and dynamics of water.

Lawrie Skinner gained his Ph.D. in physics from Bristol University studying novel aluminate glasses using neutron and synchrotron X-ray scattering under the supervision of Dr. Adrian Barnes. He then applied these pair distribution function techniques to cementitious materials as a postdoctoral associate at the University of California Berkeley. In 2010 he moved to Stony Brook University initially as a postdoctoral associate, and was later promoted to research assistant professor. At Stony Brook Lawrie Skinner has been combining synchrotron X-ray scattering with advanced sample environments to study important liquids such as water and molten uranium dioxide.

\section{ACKNOWLEDGMENTS}

This review was initiated during the Nordita (Nordic Institute for Theoretical Physics) scientific program "Water-the Most Anomalous Liquid". Additional financial support for this program was provided by the Royal Swedish Academy of Sciences through its Nobel Institutes for Physics and Chemistry, by the Swedish Research Council, and by the Department of Physics at Stockholm University. L.E.B.'s work was supported by the Swiss National Science Foundation through FNS Grant 200021-149847 and by the French state funds managed by ANR within the Blanc International program PACS under reference ANR-13-IS04-0006-01 and the Investissements d'Avenir program under reference ANR-11-IDEX-0004-02, and more specifically within the framework of the Cluster of Excellence 
MATériaux Interfaces Surfaces Environnement (MATISSE) led by Sorbonne Universités. T.L. is grateful for funding to the European Research Council (ERC Starting Grant SULIWA), the Austrian Science Fund FWF (bilateral project I1392) and the Alexander von Humboldt Foundation (Bessel award). L.E.B. and A.P. kindly thank Umbertoluca Ranieri for his help in figure editing.

\section{ABBREVIATIONS}

$\begin{array}{ll}\text { APS } & \text { Advanced Photon Source } \\ \text { ASW } & \text { amorphous solid water } \\ \text { DNA } & \text { DNA } \\ \text { ESRF } & \text { European Synchrotron Radiation Facility } \\ \text { ESS } & \text { European Spallation Source } \\ \text { HBB } & \text { hydrogen-bond bending } \\ \text { HBS } & \text { hydrogen-bond stretching } \\ \text { HDA } & \text { high-density amorphous ice } \\ \text { HGW } & \text { hyperquenched glassy water } \\ \text { IINS } & \text { incoherent inelastic neutron scattering } \\ \text { INS } & \text { inelastic neutron scattering } \\ \text { IXS } & \text { inelastic X-ray scattering } \\ \text { LCLS } & \text { Linac Coherent Light Source } \\ \text { LDA } & \text { low-density amorphous ice } \\ \text { LIB } & \text { hydrogen-bond libration } \\ \text { MBP } & \text { maltose binding protein } \\ \text { MD } & \text { molecular dynamics } \\ \text { NAGMA } & N \text {-acetyl-glycine-methylamide } \\ \text { NALMA } & N \text {-acetyl-leucine-methylamide } \\ \text { QENS } & \text { quasi-elastic neutron scattering } \\ \text { RNase } & \text { ribonuclease } \\ \text { SACLA } & \text { SPring-8 Angstrom Compact free electron LAser } \\ \text { VDOS } & \text { vibrational density of states } \\ \text { VHDA } & \text { very-high density amorphous ice }\end{array}$

\section{REFERENCES}

(1) Stanley, H. E.; Teixeira, J. Interpretation of the Unusual Behavior of $\mathrm{H} 2 \mathrm{O}$ and D2O at Low Temperatures: Tests of a Percolation Model. J. Chem. Phys. 1980, 73, 3404-3422.

(2) Teixeira, J.; Bellissent-Funel, M.-C.; Chen, S. H.; Dianoux, A. J. Experimental-Determination of the Nature of Diffusive Motions of Water-Molecules at Low-Temperatures. Phys. Rev. A: At., Mol., Opt. Phys. 1985, 31, 1913-1917.

(3) Ranieri, U.; Giura, P.; Gorelli, F. A.; Santoro, M.; Klotz, S.; Gillet, P.; Paolasini, L.; Koza, M. M.; Bove, L. E. Dynamical Crossover in Hot Dense Water: the Hydrogen Bond Role. J. Phys. Chem. B 2016, accepted.

(4) Amann-Winkel, K.; Gainaru, C.; Handle, P. H.; Seidl, M.; Nelson, H.; Bohmer, R.; Loerting, T. Water's Second Glass Transition. Proc. Natl. Acad. Sci. U. S. A. 2013, 110, 17720-17725.

(5) Head-Gordon, T.; Hura, G. Water Structure from Scattering Experiments and Simulation. Chem. Rev. 2002, 102, 2651-2669.

(6) Bakker, H. J.; Skinner, J. L. Vibrational Spectroscopy as a Probe of Structure and Dynamics in Liquid Water. Chem. Rev. 2010, 110, 14981517.

(7) Soper, A. K. Supercooled Water: Continuous Trends. Nat. Mater. 2014, 13, 671-673.

(8) Angell, C. A. Supercooled Water: Two Phases? Nat. Mater. 2014, $13,673-675$

(9) Finney, J. L. Water? What's so Special About It? Philos. Trans. R. Soc., B 2004, 359, 1145-1163.

(10) Teixeira, J. Recent Experimental Aspects of the Structure and Dynamics of Liquid and Supercooled Water. Mol. Phys. 2012, 110, 249258.

(11) Skinner, L. B.; Huang, C.; Schlesinger, D.; Pettersson, L. G.; Nilsson, A.; Benmore, C. J. Benchmark Oxygen-Oxygen Pair-
Distribution Function of Ambient Water from X-Ray Diffraction Measurements with a Wide Q-Range. J. Chem. Phys. 2013, 138, 074506. (12) Sellberg, J. A.; Huang, C.; McQueen, T. A.; Loh, N. D.; Laksmono, H.; Schlesinger, D.; Sierra, R. G.; Nordlund, D.; Hampton, C. Y.; Starodub, D.; et al. Ultrafast X-Ray Probing of Water Structure Below the Homogeneous Ice Nucleation Temperature. Nature 2014, 510, 381-384.

(13) Loerting, T.; Winkel, K.; Seidl, M.; Bauer, M.; Mitterdorfer, C.; Handle, P. H.; Salzmann, C. G.; Mayer, E.; Finney, J. L.; Bowron, D. T. How Many Amorphous Ices Are There? Phys. Chem. Chem. Phys. 2011, 13, 8783-8794.

(14) Bove, L. E.; Klotz, S.; Strassle, T.; Koza, M.; Teixeira, J.; Saitta, A. M. Translational and Rotational Diffusion in Water in the Gigapascal Range. Phys. Rev. Lett. 2013, 111, 185901.

(15) Fanetti, S.; Pagliai, M.; Citroni, M.; Lapini, A.; Scandolo, S.; Righini, R.; Bini, R. Connecting the Water Phase Diagram to the Metastable Domain: High-Pressure Studies in the Supercooled Regime. J. Phys. Chem. Lett. 2014, 5, 3804-3809.

(16) Paciaroni, A.; Nibali, V. C.; Orecchini, A.; Petrillo, C.; Haertlein, M.; Moulin, M.; Tarek, M.; D’Angelo, G.; Sacchetti, F. Vibrational Excitations of Proteins and their Hydration Water in the Far-Infrared Range. Chem. Phys. 2013, 424, 80-83.

(17) Russo, D.; Teixeira, J.; Kneller, L.; Copley, J. R. D.; Ollivier, J.; Perticaroli, S.; Pellegrini, E.; Gonzalez, M. A. Vibrational Density of States of Hydration Water at Biomolecular Sites: Hydrophobicity Promotes Low Density Amorphous Ice Behavior. J. Am. Chem. Soc. 2011, 133, 4882-4888.

(18) Mallamace, F.; Corsaro, C.; Chen, S. H.; Stanley, H. E. In Liquid Polymorphism; Stanley, H. E., Ed.; Advances in Chemical Physics 152; John Wiley \& Sons: Hoboken, NJ, USA, 2013.

(19) Lovesey, S. W. Theory of Neutron Scattering from Condensed Matter, 3rd ed.; Clarendon Press: Oxford, U.K., 1987.

(20) Morgan, J.; Warren, B. X-Ray Analysis of the Structure of Water. J. Chem. Phys. 1938, 6, 666-673.

(21) Wernet, P.; Nordlund, D.; Bergmann, U.; Cavalleri, M.; Odelius, M.; Ogasawara, H.; Naslund, L. A.; Hirsch, T. K.; Ojamae, L.; Glatzel, P.; et al. The Structure of the First Coordination Shell in Liquid Water. Science 2004, 304, 995-999.

(22) Clark, G. N. I.; Cappa, C. D.; Smith, J. D.; Saykally, R. J.; HeadGordon, T. Invited Topical Review: The Structure of Ambient Water. Mol. Phys. 2010, 108, 1415-1433.

(23) Leetmaa, M.; Wikfeldt, K. T.; Ljungberg, M. P.; Odelius, M.; Swenson, J.; Nilsson, A.; Pettersson, L. G. Diffraction and IR/Raman Data do not Prove Tetrahedral Water. J. Chem. Phys. 2008, 129, 084502.

(24) Giguere, P. A. Bifurcated Hydrogen-Bonds in Water. J. Raman Spectrosc. 1984, 15, 354-359.

(25) Sciortino, F.; Geiger, A.; Stanley, H. E. Effect of Defects on Molecular Mobility in Liquid Water. Nature 1991, 354, 218-221.

(26) Narten, A. H.; Thiessen, W. E.; Blum, L. Atom Pair Distribution Functions of Liquid Water at $25^{\circ} \mathrm{C}$ from Neutron Diffraction. Science 1982, 217, 1033-1034.

(27) Soper, A. K. The Radial Distribution Functions of Water as Derived from Radiation Total Scattering Experiments: Is There Anything We Can Say for Sure? ISRN Phys. Chem. 2013, 2013, 279463.

(28) Dianoux, A.-J.; Lander, G. Neutron Data Booklet, 2nd ed.; Old City Publishing: Philadelphia, PA, USA, 2003.

(29) Fischer, H. E.; Simonson, J. M.; Neuefeind, J. C.; Lemmel, H.; Rauch, H.; Zeidler, A.; Salmon, P. S. The Bound Coherent Neutron Scattering Lengths of the Oxygen Isotopes. J. Phys.: Condens. Matter 2012, 24, 505105.

(30) Fischer, H. E.; Barnes, A. C.; Salmon, P. S. Neutron and X-ray Diffraction Studies of Liquids and Glasses. Rep. Prog. Phys. 2006, 69, 233-299.

(31) Dore, J. C.; Garawi, M.; Bellissent-Funel, M.-C. Neutron Diffraction Studies of the Structure of Water at Ambient Temperatures, Revisited [A Review of Past Developments and Current Problems]. Mol. Phys. 2004, 102, 2015-2035. 
(32) Hart, R. T.; Benmore, C. J.; Neuefeind, J.; Kohara, S.; Tomberli, B.; Egelstaff, P. A. Temperature Dependence of Isotopic Quantum Effects in Water. Phys. Rev. Lett. 2005, 94, 047801.

(33) Hart, R. T.; Mei, Q.; Benmore, C. J.; Neuefeind, J. C.; Turner, J. F.; Dolgos, M.; Tomberli, B.; Egelstaff, P. A. Isotope Quantum Effects in Water Around the Freezing Point. J. Chem. Phys. 2006, 124, 134505.

(34) Rottger, K.; Endriss, A.; Ihringer, J.; Doyle, S.; Kuhs, W. F. LatticeConstants and Thermal-Expansion of $\mathrm{H} 2 \mathrm{O}$ and D2O Ice Ih Between 10 and 265 K. Acta Crystallogr., Sect. B: Struct. Sci. 1994, 50, 644-648.

(35) Zeidler, A.; Salmon, P. S.; Fischer, H. E.; Neuefeind, J. C.; Simonson, J. M.; Lemmel, H.; Rauch, H.; Markland, T. E. Oxygen as a Site Specific Probe of the Structure of Water and Oxide Materials. Phys. Rev. Lett. 2011, 107, 145501.

(36) Zeidler, A.; Salmon, P. S.; Fischer, H. E.; Neuefeind, J. C.; Simonson, J. M.; Markland, T. E. Isotope Effects in Water as Investigated by Neutron Diffraction and Path Integral Molecular Dynamics. J. Phys.: Condens. Matter 2012, 24, 284126.

(37) Soper, A. K. Joint Structure Refinement of X-Ray and Neutron Diffraction Data on Disordered Materials: Application to Liquid Water. J. Phys.: Condens. Matter 2007, 19, 335206.

(38) Sorenson, J. M.; Hura, G.; Glaeser, R. M.; Head-Gordon, T. What can X-Ray Scattering Tell Us About the Radial Distribution Functions of Water? J. Chem. Phys. 2000, 113, 9149-9161.

(39) Wang, J.; Tripathi, A. N.; Smith, V. H. Chemical Binding and Electron Correlation Effects in X-Ray and High Energy Electron Scattering. J. Chem. Phys. 1994, 101, 4842-4854.

(40) Skinner, L. B.; Benmore, C. J.; Neuefeind, J. C.; Parise, J. B. The Structure of Water Around the Compressibility Minimum. J. Chem. Phys. 2014, 141, 214507.

(41) Neuefeind, J.; Benmore, C. Formalism for the Determination of Structural Isotope Effects with Neutrons. Nucl. Instrum. Methods Phys. Res., Sect. A 2009, 600, 257-259.

(42) Soper, A. K.; Benmore, C. J. Quantum Differences Between Heavy and Light Water. Phys. Rev. Lett. 2008, 101, 065502.

(43) Bergmann, U.; Nordlund, D.; Wernet, P.; Odelius, M.; Pettersson, L. G. M.; Nilsson, A. Isotope Effects in Liquid Water Probed by X-ray Raman Spectroscopy. Phys. Rev. B: Condens. Matter Mater. Phys. 2007, 76, 024202

(44) Koch, M. H.; Vachette, P.; Svergun, D. I. Small-Angle Scattering: a View on the Properties, Structures and Structural Changes of Biological Macromolecules in Solution. Q. Rev. Biophys. 2003, 36, 147-227.

(45) Bosio, L.; Teixeira, J.; Bellissent-Funel, M.-C. Enhanced Density Fluctuations in Water Analyzed by Neutron Scattering. Phys. Rev. A: At., Mol., Opt. Phys. 1989, 39, 6612-6613.

(46) Bosio, L.; Teixeira, J.; Stanley, H. E. Enhanced Density Fluctuations in Supercooled H2O, D2O, and Ethanol-Water Solutions: Evidence from Small-Angle X-Ray Scattering. Phys. Rev. Lett. 1981, 46, 597.

(47) Xie, Y.; Ludwig, K. F., Jr.; Morales, G.; Hare, D. E.; Sorensen, C. M. Noncritical Behavior of Density Fluctuations in Supercooled Water. Phys. Rev. Lett. 1993, 71, 2050-2053.

(48) Michielsen, J. C.; Bot, A.; van der Elsken, J. Small-Angle X-Ray Scattering from Supercooled Water. Phys. Rev. A: At., Mol., Opt. Phys. 1988, 38, 6439-6441.

(49) Dings, J.; Michielsen, J. C.; van der Elsken, J. Equilibrium and Nonequilibrium Contributions to X-ray Scattering from Supercooled Water. Phys. Rev. A: At., Mol., Opt. Phys. 1992, 45, 5731-5733.

(50) Smolsky, I. L.; Liu, P.; Niebuhr, M.; Ito, K.; Weiss, T. M.; Tsuruta, H. Biological Small-Angle X-Ray Scattering Facility at the Stanford Synchrotron Radiation Laboratory. J. Appl. Crystallogr. 2007, 40, s453s458.

(51) Huang, C.; Wikfeldt, K. T.; Tokushima, T.; Nordlund, D.; Harada, Y.; Bergmann, U.; Niebuhr, M.; Weiss, T. M.; Horikawa, Y.; Leetmaa, M.; et al. The Inhomogeneous Structure of Water at Ambient Conditions. Proc. Natl. Acad. Sci. U. S. A. 2009, 106, 15214-15218.

(52) Huang, C.; Weiss, T. M.; Nordlund, D.; Wikfeldt, K. T.; Pettersson, L. G.; Nilsson, A. Increasing Correlation Length in Bulk Supercooled H2O, D2O, and $\mathrm{NaCl}$ Solution Determined from Small Angle X-ray Scattering. J. Chem. Phys. 2010, 133, 134504.
(53) Nilsson, A.; Huang, C. C.; Pettersson, L. G. M. Fluctuations in Ambient Water. J. Mol. Liq. 2012, 176, 2-16.

(54) Hendricks, R.; Mardon, P.; Shaffer, L. X-ray Zero-Angle Scattering Cross Section of Water. J. Chem. Phys. 1974, 61, 319-322.

(55) Kell, G. S. Isothermal Compressibility of Liquid Water at 1 Atm. J. Chem. Eng. Data 1970, 15, 119.

(56) Huang, C.; Wikfeldt, K. T.; Tokushima, T.; Nordlund, D.; Harada, Y.; Bergmann, U.; Niebuhr, M.; Weiss, T. M.; Horikawa, Y.; Leetmaa, M.; et al. Reply to Soper et al.: Fluctuations in Water Around a Bimodal Distribution of Local Hydrogen-Bonded Structural Motifs. Proc. Natl. Acad. Sci. U. S. A. 2010, 107, E45-E45.

(57) Nilsson, A.; Pettersson, L. G. M. Perspective on the Structure of Liquid Water. Chem. Phys. 2011, 389, 1-34.

(58) Overduin, S. D.; Patey, G. N. Understanding the Structure Factor and Isothermal Compressibility of Ambient Water in Terms of Local Structural Environments. J. Phys. Chem. B 2012, 116, 12014-12020.

(59) Wikfeldt, K. T.; Huang, C.; Nilsson, A.; Pettersson, L. G. Enhanced Small-Angle Scattering Connected to the Widom Line in Simulations of Supercooled Water. J. Chem. Phys. 2011, 134, 214506.

(60) Clark, G. N.; Hura, G. L.; Teixeira, J.; Soper, A. K.; Head-Gordon, T. Small-Angle Scattering and the Structure of Ambient Liquid Water. Proc. Natl. Acad. Sci. U. S. A. 2010, 107, 14003-14007.

(61) Soper, A.; Teixeira, J.; Head-Gordon, T. Is Ambient Water Inhomogeneous on the Nanometer-Length Scale? Proc. Natl. Acad. Sci. U. S. A. 2010, 107, E44.

(62) Soper, A. K. Recent Water Myths. Pure Appl. Chem. 2010, 82, 1855-1867.

(63) Abascal, J. L.; Vega, C. A General Purpose Model for the Condensed Phases of Water: TIP4P/2005. J. Chem. Phys. 2005, 123, 234505.

(64) Guinier, A.; Fournet, G. Small Angle Scattering of X-rays; John Wiley \& Sons: 1955.

(65) Moore, E. B.; Molinero, V. Growing Correlation Length in Supercooled Water. J. Chem. Phys. 2009, 130, 244505.

(66) Kühne, T. D.; Khaliullin, R. Z. Electronic Signature of the Instantaneous Asymmetry in the First Coordination Shell of Liquid Water. Nat. Commun. 2013, 4, 1450.

(67) Berendsen, H. J. C.; Grigera, J. R.; Straatsma, T. P. The Missing Term in Effective Pair Potentials. J. Phys. Chem. 1987, 91, 6269-6271.

(68) Wang, L. P.; Head-Gordon, T.; Ponder, J. W.; Ren, P.; Chodera, J. D.; Eastman, P. K.; Martinez, T. J.; Pande, V. S. Systematic Improvement of a Classical Molecular Model of Water. J. Phys. Chem. B 2013, 117, 9956-9972.

(69) Amann-Winkel, K.; Böhmer, R.; Fujara, F.; Gainaru, C.; Geil, B.; Loerting, T. Colloquium: Water's Controversial Glass Transitions. Rev. Mod. Phys. 2016, 88, 011002.

(70) Bowron, D.; Finney, J.; Hallbrucker, A.; Kohl, I.; Loerting, T.; Mayer, E.; Soper, A. The Local and Intermediate Range Structures of the Five Amorphous Ices at $80 \mathrm{~K}$ and Ambient Pressure: A Faber-Ziman and Bhatia-Thornton Analysis. J. Chem. Phys. 2006, 125, 194502.

(71) Burton, E. F.; Oliver, W. F. X-ray Diffraction Patterns of Ice. Nature 1935, 135, 505.

(72) Kohl, I.; Bachmann, L.; Hallbrucker, A.; Mayer, E.; Loerting, T. Liquid-Like Relaxation in Hyperquenched Water at $\leq 140 \mathrm{~K}$. Phys. Chem. Chem. Phys. 2005, 7, 3210-3220.

(73) Mishima, O.; Calvert, L.; Whalley, E. An Apparently First-Order Transition Between Two Amorphous Phases of Ice Induced by Pressure. Nature 1985, 314, 76-78.

(74) Loerting, T.; Bauer, M.; Kohl, I.; Watschinger, K.; Winkel, K.; Mayer, E. Cryoflotation: Densities of Amorphous and Crystalline Ices. J. Phys. Chem. B 2011, 115, 14167-14175.

(75) Tse, J. S.; Klug, D. D. Pressure Amorphized Ices - An Atomistic Perspective. Phys. Chem. Chem. Phys. 2012, 14, 8255-8263.

(76) Tse, J.; Klug, D.; Tulk, C.; Swainson, I.; Svensson, E.; Loong, C.K.; Shpakov, V.; Belosludov, V.; Belosludov, R.; Kawazoe, Y. The Mechanisms for Pressure-Induced Amorphization of Ice Ih. Nature 1999, 400, 647-649.

(77) Dowell, L. G.; Rinfret, A. P. Low-Temperature Forms of Ice as Studied by X-ray Diffraction. Nature 1960, 188, 1144-1148. 
(78) Venkatesh, C. G.; Rice, S. A.; Narten, A. H. Amorphous Solid Water - X-Ray-Diffraction Study. Science 1974, 186, 927-928.

(79) Hallbrucker, A.; Mayer, E.; O'Mard, L. P.; Dore, J. C.; Chieux, P. Structural Characterization of Hyperquenched Glassy Water and VaporDeposited Amorphous Ice. Phys. Lett. A 1991, 159, 406-410.

(80) Bellissent-Funel, M.-C.; Bosio, L.; Hallbrucker, A.; Mayer, E.; Srididorbez, R. X-ray and Neutron-Scattering Studies of the Structure of Hyperchenched Glassy Water. J. Chem. Phys. 1992, 97, 1282-1286.

(81) Floriano, M. A.; Whalley, E.; Svensson, E. C.; Sears, V. F. Structure of High-Density Amorphous Ice by Neutron-Diffraction. Phys. Rev. Lett. 1986, 57, 3062-3064.

(82) Bellissent-Funel, M.-C.; Teixeira, J.; Bosio, L. Structure of HighDensity Amorphous Water. 2. Neutron-Scattering Study. J. Chem. Phys. 1987, 87, 2231-2235.

(83) Bizid, A.; Bosio, L.; Defrain, A.; Oumezzine, M. Structure of HighDensity Amorphous Water. 1. X-ray-Diffraction Study. J. Chem. Phys. 1987, 87, 2225-2230.

(84) Winkel, K.; Bowron, D. T.; Loerting, T.; Mayer, E.; Finney, J. L. Relaxation Effects in Low Density Amorphous Ice: Two Distinct Structural States Observed by Neutron Diffraction. J. Chem. Phys. 2009, $130,204502$.

(85) Handa, Y. P.; Mishima, O.; Whalley, E. High-Density Amorphous Ice. III. Thermal Properties. J. Chem. Phys. 1986, 84, 2766-2770.

(86) Koza, M. M.; Schober, H.; Fischer, H. E.; Hansen, T.; Fujara, F. Kinetics of the High- to Low-Density Amorphous Water Transition. J. Phys.: Condens. Matter 2003, 15, 321-332.

(87) Nelmes, R. J.; Loveday, J. S.; Strassle, T.; Bull, C. L.; Guthrie, M.; Hamel, G.; Klotz, S. Annealed High-Density Amorphous Ice Under Pressure. Nat. Phys. 2006, 2, 414-418.

(88) Winkel, K.; Mayer, E.; Loerting, T. Equilibrated High-Density Amorphous Ice and its First-Order Transition to the Low-Density form. J. Phys. Chem. B 2011, 115, 14141-14148.

(89) Tulk, C. A.; Benmore, C. J.; Urquidi, J.; Klug, D. D.; Neuefeind, J.; Tomberli, B.; Egelstaff, P. A. Structural Studies of Several Distinct Metastable Forms of Amorphous Ice. Science 2002, 297, 1320-1323.

(90) Elsaesser, M. S.; Winkel, K.; Mayer, E.; Loerting, T. Reversibility and Isotope Effect of the Calorimetric Glass $\rightarrow$ Liquid Transition of Low-Density Amorphous Ice. Phys. Chem. Chem. Phys. 2010, 12, 708712.

(91) Amann-Winkel, K.; Low, F.; Handle, P. H.; Knoll, W.; Peters, J.; Geil, B.; Fujara, F.; Loerting, T. Limits of Metastability in Amorphous Ices: the Neutron Scattering Debye-Waller Factor. Phys. Chem. Chem. Phys. 2012, 14, 16386-16391.

(92) Seidl, M.; Amann-Winkel, K.; Handle, P. H.; Zifferer, G.; Loerting, T. From Parallel to Single Crystallization Kinetics in HighDensity Amorphous Ice. Phys. Rev. B: Condens. Matter Mater. Phys. 2013, $88,174105$.

(93) Seidl, M.; Fayter, A.; Stern, J. N.; Zifferer, G.; Loerting, T. Shrinking water's no man's land by lifting its low-temperature boundary. Phys. Rev. B: Condens. Matter Mater. Phys. 2015, 91, 144201.

(94) Koza, M. M.; Geil, B.; Winkel, K.; Kohler, C.; Czeschka, F.; Scheuermann, M.; Schober, H.; Hansen, T. Nature of Amorphous Polymorphism of Water. Phys. Rev. Lett. 2005, 94, 125506.

(95) Koza, M. M.; Hansen, T.; May, R. P.; Schober, H. Link Between the Diversity, Heterogeneity and Kinetic Properties of Amorphous Ice Structures. J. Non-Cryst. Solids 2006, 352, 4988-4993.

(96) Koza, M. M.; May, R. P.; Schober, H. On the Heterogeneous Character of Water's Amorphous Polymorphism. J. Appl. Crystallogr. 2007, 40, s517-s521.

(97) Loerting, T.; Brazhkin, V. V.; Morishita, T. In Advances in Chemical Physics; Rice, S. A., Ed.; John Wiley \& Sons: 2009; Vol. 143.

(98) Schober, H.; Koza, M. M.; Tolle, A.; Masciovecchio, C.; Sette, F.; Fujara, F. Crystal-like High Frequency Phonons in the Amorphous Phases of Solid Water. Phys. Rev. Lett. 2000, 85, 4100-4103.

(99) Johari, G.; Hallbrucker, A.; Mayer, E. The Glass-Liquid Transition of Hyperquenched Water. Nature 1987, 330, 552-553.

(100) Andersson, O. Glass-Liquid Transition of Water at High Pressure. Proc. Natl. Acad. Sci. U. S. A. 2011, 108, 11013-11016.
(101) Loerting, T.; Fuentes-Landete, V.; Handle, P. H.; Seidl, M.; Amann-Winkel, K.; Gainaru, C.; Bohmer, R. The Glass Transition in High-Density Amorphous Ice. J. Non-Cryst. Solids 2015, 407, 423-430. (102) Fujara, F.; Petry, W.; Diehl, R. M.; Schnauss, W.; Sillescu, H. Localized Motion in Supercooled Glycerol as Measured by H-2-NMR Spin-Lattice Relaxation and Incoherent Neutron-Scattering. Europhys. Lett. 1991, 14, 563-568.

(103) Geil, B.; Koza, M. M.; Fujara, F.; Schober, H.; Natali, F. Absence of Fast Precursor Dynamics of Low-Density Amorphous Ice Around Its Hypothetical Glass Transition Temperature. Phys. Chem. Chem. Phys. 2004, 6, 677-679.

(104) Chen, S.-H.; Teixeira, J.; Nicklow, R. Incoherent Quasielastic Neutron Scattering from Water in Supercooled Regime. Phys. Rev. A: At., Mol., Opt. Phys. 1982, 26, 3477.

(105) Cunsolo, A.; Orecchini, A.; Petrillo, C.; Sacchetti, F. Quasielastic Neutron Scattering Investigation of the Pressure Dependence of Molecular Motions in Liquid Water. J. Chem. Phys. 2006, 124, 084503.

(106) Tassaing, T.; Bellissent-Funel, M.-C. The Dynamics of Supercritical Water: A Quasielastic Incoherent Neutron Scattering Study. J. Chem. Phys. 2000, 113, 3332-3337.

(107) Cunsolo, A.; Orecchini, A.; Petrillo, C.; Sacchetti, F. Interplay between Microscopic Diffusion and Local Structure of Liquid Water. J. Phys. Chem. B 2010, 114, 16713-16717.

(108) Lang, E.; Lüdemann, H.-D. High Pressure O-17 Longitudinal Relaxation Time Studies in Supercooled H20 and D20. Ber. Bunsenges. Phys. Chem. 1981, 85, 603-611.

(109) Lang, E. W.; Ludemann, H. D. Anomalies of Liquid Water. Angew. Chem., Int. Ed. Engl. 1982, 21, 315-329.

(110) Qvist, J.; Schober, H.; Halle, B. Structural Dynamics of Supercooled Water from Quasielastic Neutron Scattering and Molecular Simulations. J. Chem. Phys. 2011, 134, 144508.

(111) Bergman, R.; Swenson, J. Dynamics of Supercooled Water in Confined Geometry. Nature 2000, 403, 283-286.

(112) Choi, C.; Bharatam, J.; Geilikman, M.; Dusseault, M. B.; Pintar, M. M. Relaxation of Water Proton Spins by Paramagnetic Spin-Label Molecules in Porous Glass. J. Appl. Phys. 1997, 82, 4461-4464.

(113) Hoptner, W.; Vonschutz, J. U.; Wolf, H. C. Proton SpinRelaxation Study of the Polymerization of Diacetylene in a SingleCrystal. J. Polym. Sci., Polym. Phys. Ed. 1980, 18, 469-478.

(114) Bove, L. E.; Klotz, S.; Paciaroni, A.; Sacchetti, F. Anomalous Proton Dynamics in Ice at Low Temperatures. Phys. Rev. Lett. 2009, 103, 165901.

(115) Drechsel-Grau, C.; Marx, D. Quantum Simulation of Collective Proton Tunneling in Hexagonal Ice Crystals. Phys. Rev. Lett. 2014, 112, 148302.

(116) Drechsel-Grau, C.; Marx, D. Exceptional Isotopic-Substitution Effect: Breakdown of Collective Proton Tunneling in Hexagonal Ice due to Partial Deuteration. Angew. Chem., Int. Ed. 2014, 53, 10937-10940.

(117) Ludwig, R.; Weinhold, F.; Farrar, T. C. Experimental and Theoretical Determination of the Temperature-Dependence of Deuteron and Oxygen Quadrupole Coupling-Constants of Liquid Water. J. Chem. Phys. 1995, 103, 6941-6950.

(118) Qvist, J.; Mattea, C.; Sunde, E. P.; Halle, B. Rotational Dynamics in Supercooled Water from Nuclear Spin Relaxation and Molecular Simulations. J. Chem. Phys. 2012, 136, 204505.

(119) Laage, D. Reinterpretation of the Liquid Water Quasi-Elastic Neutron Scattering Spectra Based on a Nondiffusive Jump Reorientation Mechanism. J. Phys. Chem. B 2009, 113, 2684-2687.

(120) Laage, D.; Hynes, J. T. On the Molecular Mechanism of Water Reorientation. J. Phys. Chem. B 2008, 112, 14230-14242.

(121) Sposito, G. Single-Particle Motions in Liquid Water. II. The Hydrodynamic Model. J. Chem. Phys. 1981, 74, 6943-6949.

(122) Fanetti, S.; Lapini, A.; Pagliai, M.; Citroni, M.; Di Donato, M.; Scandolo, S.; Righini, R.; Bini, R. Structure and Dynamics of LowDensity and High-Density Liquid Water at High Pressure. J. Phys. Chem. Lett. 2014, 5, 235-240.

(123) Bée, M. Quasielastic Neutron Scattering; Adam Hilger: Bristol, U.K., 1988. 
(124) Klotz, S.; Strassle, T.; Bove, L. E. Quasi-elastic Neutron Scattering in the Multi-GPa Range and its Application to Liquid Water. Appl. Phys. Lett. 2013, 103, 193504.

(125) Jonas, J.; Defried, T.; Wilbur, D. J. Molecular Motions in Compressed Liquid Water. J. Chem. Phys. 1976, 65, 582-588.

(126) Wilbur, D. J.; Defries, T.; Jonas, J. Self-Diffusion in Compressed Liquid Heavy-Water. J. Chem. Phys. 1976, 65, 1783-1786.

(127) Krynicki, K.; Green, C. D.; Sawyer, D. W. Pressure and Temperature-Dependence of Self-Diffusion in Water. Faraday Discuss. Chem. Soc. 1978, 66, 199-208.

(128) Simpson, J. H.; Carr, H. Y. Diffusion and Nuclear Spin Relaxation in Water. Phys. Rev. 1958, 111, 1201-1202.

(129) Gillen, K. T.; Douglass, D. C.; Hoch, J. R. Self-Diffusion in Liquid Water to -31 Degree C. J. Chem. Phys. 1972, 57, 5117-5119.

(130) Agmon, N. Tetrahedral Displacement: The Molecular Mechanism behind the Debye Relaxation in Water. J. Phys. Chem. 1996, 100, 1072-1080.

(131) Harris, K. R.; Newitt, P. J. Self-Diffusion of Water at Low Temperatures and High Pressure. J. Chem. Eng. Data 1997, 42, 346348.

(132) Price, W. S.; Ide, H.; Arata, Y. Self-Diffusion of Supercooled Water to $238 \mathrm{~K}$ Using PGSE NMR Diffusion Measurements. J. Phys. Chem. A 1999, 103, 448-450.

(133) Prielmeier, F. X.; Lang, E. W.; Speedy, R. J.; Ludemann, H. D. Diffusion in Supercooled Water to 300-MPA. Phys. Rev. Lett. 1987, 59, 1128-1131.

(134) Prielmeier, F. X.; Lang, E. W.; Speedy, R. J.; Lüdemann, H. D. The Pressure Dependence of Self Diffusion in Supercooled Light and Heavy Water. Ber. Bunsenges. Phys. Chem. 1988, 92, 1111-1117.

(135) Cunsolo, A.; Orecchini, A.; Petrillo, C.; Sacchetti, F. Pressure Evolution of Microscopic Diffusion in Liquid Water. J. Neutron Res. 2006, 14, 309-315.

(136) Krynicki, K. Proton Spin-Lattice Relaxation in Pure Water Between Zero Degrees C and 100 Degrees C. Physica 1966, 32, 167.

(137) Teixeira, J.; Luzar, A.; Longeville, S. Dynamics of Hydrogen Bonds: How to Probe their Role in the Unusual Properties of Liquid Water. J. Phys.: Condens. Matter 2006, 18, S2353-S2362.

(138) Richter, D.; Monkenbusch, M.; Arbe, A.; Colmenero, J.; Farago, B. Dynamic Structure Factors Due to Relaxation Processes in GlassForming Polymers. Phys. B 1997, 241-243, 1005-1012.

(139) Bellissent-Funel, M. C.; Bosio, L. A Neutron Scattering Study of Liquid D2O under Pressure and at Various Temperatures. J. Chem. Phys. 1995, 102, 3727-3735.

(140) Saitta, A. M.; Strassle, T.; Klotz, S. Structural Properties of the Amorphous Ices: An Analysis in Terms of Distance-Ranked Neighbors and Angular Correlations. J. Phys. Chem. B 2006, 110, 3595-3603.

(141) Strassle, T.; Saitta, A. M.; Le Godec, Y.; Hamel, G.; Klotz, S.; Loveday, J. S.; Nelmes, R. J. Structure of Dense Liquid Water by Neutron Scattering to $6.5 \mathrm{GPa}$ and $670 \mathrm{~K}$. Phys. Rev. Lett. 2006, 96, 067801.

(142) Saitta, A. M.; Datchi, F. Structure and Phase Diagram of HighDensity Water: The Role of Interstitial Molecules. Phys. Rev. E: Stat. Phys., Plasmas, Fluids, Relat. Interdiscip. Top. 2003, 67, 020201.

(143) Wang, Z.; Liu, K. H.; Le, P.; Li, M.; Chiang, W. S.; Leao, J. B.; Copley, J. R.; Tyagi, M.; Podlesnyak, A.; Kolesnikov, A. I.; et al. Boson Peak in Deeply Cooled Confined Water: A Possible Way to Explore the Existence of the Liquid-to-Liquid Transition in Water. Phys. Rev. Lett. 2014, 112, 237802.

(144) Price, D. L.; Sköld, K. Methods in Experimental Physics. Neutron Scattering; Academic Press: Orlando, FL, USA, 1986.

(145) Chen, S.-H.; Teixeira, J.; Nicklow, R. In Advances in Chemical Physics; Prigogine, I., Rice, S. A., Eds.; John Wiley \& Sons: Chichester, U.K., 1986; Vol. 64

(146) Bellissent-Funel, M.-C.; Teixeira, J. Dynamics of Water Studied by Coherent and Incoherent Inelastic Neutron Scattering. J. Mol. Struct. 1991, 250, 213-230.

(147) Walrafen, G. In Water a Comprehensive Treatise; Franks, F., Ed.; Plenum Press: New York, 1972; Vol. 1.
(148) Bertie, J.; Whalley, E. Optical Spectra of Orientationally Disordered Crystals. II. Infrared Spectrum of Ice Ih and Ice Ic from 360 to $50 \mathrm{~cm}^{-1}$. J. Chem. Phys. 1967, 46, 1271-1284.

(149) Heyden, M.; Sun, J.; Funkner, S.; Mathias, G.; Forbert, H.; Havenith, M.; Marx, D. Dissecting the THz Spectrum of Liquid Water from First Principles via Correlations in Time and Space. Proc. Natl. Acad. Sci. U. S. A. 2010, 107, 12068-12073.

(150) Walrafen, G. Raman Spectrum of Water: Transverse and Longitudinal Acoustic Modes Below. apprxeq. $300 \mathrm{~cm}-1$ and Optic Modes Above. apprxeq. $300 \mathrm{~cm}-1$. J. Phys. Chem. 1990, 94, 2237-2239.

(151) Toukan, K.; Ricci, M. A.; Chen, S. H.; Loong, C. K.; Price, D. L.; Teixeira, J. Neutron-Scattering Measurements of Wave-Vector-Dependent Hydrogen Density of States in Liquid Water. Phys. Rev. A: At., Mol., Opt. Phys. 1988, 37, 2580-2589.

(152) Sakamoto, M.; Johnson, R. G.; Brockhouse, B. N.; Pope, N. K. Neutron Inelastic Scattering Study of Water. J. Phys. Soc. Jpn. 1962, 17, 370.

(153) Bellissent-Funel, M.-C.; Chen, S. H.; Zanotti, J. Single-Particle Dynamics of Water Molecules in Confined Space. Phys. Rev. E: Stat. Phys., Plasmas, Fluids, Relat. Interdiscip. Top. 1995, 51, 4558-4569.

(154) Bellissent-Funel, M.-C.; Zanotti, J.; Chen, S. Slow Dynamics of Water Molecules on the Surface of Globular Proteins. Faraday Discuss. 1996, 103, 281-294.

(155) Ruffle, S. V.; Michalarias, I.; Li, J. C.; Ford, R. C. Inelastic Incoherent Neutron Scattering Studies of Water Interacting with Biological Macromolecules. J. Am. Chem. Soc. 2002, 124, 565-569.

(156) Paciaroni, A.; Orecchini, A.; Cornicchi, E.; Marconi, M.; Petrillo, C.; Haertlein, M.; Moulin, M.; Schober, H.; Tarek, M.; Sacchetti, F. Fingerprints of Amorphous Icelike Behavior in the Vibrational Density of States of Protein Hydration Water. Phys. Rev. Lett. 2008, 101, 148104.

(157) Lerbret, A.; Hedoux, A.; Annighofer, B.; Bellissent-Funel, M.-C. Influence of Pressure on the Low-Frequency Vibrational Modes of Lysozyme and Water: A Complementary Inelastic Neutron Scattering and Molecular Dynamics Simulation Study. Proteins: Struct., Funct., Genet. 2013, 81, 326-340.

(158) Li, J. C. Inelastic Neutron Scattering Studies of Hydrogen Bonding in Ices. J. Chem. Phys. 1996, 105, 6733-6755.

(159) Kolesnikov, A. I.; Li, J. C.; Parker, S. F.; Eccleston, R. S.; Loong, C. K. Vibrational Dynamics of Amorphous Ice. Phys. Rev. B: Condens. Matter Mater. Phys. 1999, 59, 3569-3578.

(160) Frick, B.; Richter, D. The Microscopic Basis of the GlassTransition in Polymers from Neutron-Scattering Studies. Science 1995, 267, 1939-1945.

(161) Tse, J. S.; Klug, D. D.; Tulk, C. A.; Svensson, E. C.; Swainson, I.; Shpakov, V. P.; Belosludov, V. R. Origin of Low-Frequency Local Vibrational Modes in High Density Amorphous Ice. Phys. Rev. Lett. 2000, 85, 3185-3188.

(162) Koza, M. M. Vibrational Dynamics of Amorphous Ice Structures Studied by High-Resolution Neutron Spectroscopy. Phys. Rev. B: Condens. Matter Mater. Phys. 2008, 78, 064303.

(163) Paciaroni, A.; Bizzarri, A. R.; Cannistraro, S. Neutron Scattering Evidence of a Boson Peak in Protein Hydration Water. Phys. Rev. E: Stat. Phys., Plasmas, Fluids, Relat. Interdiscip. Top. 1999, 60, R2476-R2479.

(164) Tarek, M.; Tobias, D. J. Single-Particle and Collective Dynamics of Protein Hydration Water: A Molecular Dynamics Study. Phys. Rev. Lett. 2002, 89, 275501.

(165) Kumar, P.; Wikfeldt, K. T.; Schlesinger, D.; Pettersson, L. G.; Stanley, H. E. The Boson Peak in Supercooled Water. Sci. Rep. 2013, 3, 1980.

(166) Chumakov, A. I.; Monaco, G.; Fontana, A.; Bosak, A.; Hermann, R. P.; Bessas, D.; Wehinger, B.; Crichton, W. A.; Krisch, M.; Ruffer, R.; et al. Role of Disorder in the Thermodynamics and Atomic Dynamics of Glasses. Phys. Rev. Lett. 2014, 112, 025502.

(167) Chen, S. H.; Toukan, K.; Loong, C. K.; Price, D. L.; Teixeira, J. Hydrogen-Bond Spectroscopy of Water by Neutron-Scattering. Phys. Rev. Lett. 1984, 53, 1360-1363.

(168) Michalarias, I.; Beta, I.; Ford, R.; Ruffle, S.; Li, J. C. Inelastic Neutron Scattering Studies of Water in DNA. Appl. Phys. A: Mater. Sci. Process. 2002, 74, s1242-s1244. 
(169) Wang, H. W.; DelloStritto, M. J.; Kumar, N.; Kolesnikov, A. I.; Kent, P. R. C.; Kubicki, J. D.; Wesolowski, D. J.; Sofo, J. O. Vibrational Density of States of Strongly H-Bonded Interfacial Water: Insights from Inelastic Neutron Scattering and Theory. J. Phys. Chem. C 2014, 118, 10805-10813.

(170) Kolesnikov, A. I.; Zanotti, J. M.; Loong, C. K.; Thiyagarajan, P.; Moravsky, A. P.; Loutfy, R. O.; Burnham, C. J. Anomalously Soft Dynamics of Water in a Nanotube: a Revelation of Nanoscale Confinement. Phys. Rev. Lett. 2004, 93, 035503.

(171) Bosi, P.; Dupre, F.; Menzingee, F.; Sacchetti, F.; Spinelli, M. Observation of Collective Excitations in Heavy Water in the $10^{8} \mathrm{~cm}^{-1}$ Momentum Range. Lett. Nuovo Cimento 1978, 21, 436-440.

(172) Teixeira, J.; Bellissent-Funel, M.-C.; Chen, S. H.; Dorner, B. Observation of New Short-Wavelength Collective Excitations in Heavy Water by Coherent Inelastic Neutron Scattering. Phys. Rev. Lett. 1985, 54, 2681-2683.

(173) Sette, F.; Ruocco, G.; Krisch, M.; Bergmann, U.; Masciovecchio, C.; Mazzacurati, V. V.; Signorelli, G.; Verbeni, R. Collective Dynamics in Water by High Energy Resolution Inelastic X-Ray Scattering. Phys. Rev. Lett. 1995, 75, 850-853.

(174) Ruocco, G.; Sette, F.; Bergmann, U.; Krisch, M.; Masciovecchio, C.; Mazzacurati, V.; Signorelli, G.; Verbeni, R. Equivalence of the Sound Velocity in Water and Ice at Mesoscopic Wavelengths. Nature 1996, 379, 521-523.

(175) Sette, F.; Ruocco, G.; Krisch, M.; Masciovecchio, C.; Verbeni, R.; Bergmann, U. Transition from Normal to Fast Sound in Liquid Water. Phys. Rev. Lett. 1996, 77, 83-86.

(176) Orecchini, A.; Paciaroni, A.; De Francesco, A.; Petrillo, C.; Sacchetti, F. Collective Dynamics of Protein Hydration Water by Brillouin Neutron Spectroscopy. J. Am. Chem. Soc. 2009, 131, 46644669.

(177) Cornicchi, E.; Sebastiani, F.; De Francesco, A.; Orecchini, A.; Paciaroni, A.; Petrillo, C.; Sacchetti, F. Collective Density Fluctuations of DNA Hydration Water in the Time-Window below 1 ps. J. Chem. Phys. 2011, 135, 025101.

(178) Sacchetti, F.; Suck, J. B.; Petrillo, C.; Dorner, B. Brillouin Neutron Scattering in Heavy Water: Evidence for Two-Mode Collective Dynamics. Phys. Rev. E 2004, 69, 061203.

(179) Berne, B. J.; Pecora, R. Dynamic Light Scattering: With Applications to Chemistry, Biology and Physics; Dover Publications: Mineola, NY, USA, 2000.

(180) Monaco, G.; Cunsolo, A.; Ruocco, G.; Sette, F. Viscoelastic Behavior of Water in the Terahertz-Frequency Range: An Inelastic XRay Scattering Study. Phys. Rev. E: Stat. Phys., Plasmas, Fluids, Relat. Interdiscip. Top. 1999, 60, 5505-5521.

(181) Cunsolo, A. The THz Spectrum of Density Fluctuations of Water: The Viscoelastic Regime. Adv. Condens. Matter Phys. 2015, 2015, 137435.

(182) Bellissent-Funel, M.-C.; Teixeira, J.; Chen, S. H.; Dorner, B.; Middendorf, H. D.; Crespi, H. L. Low-Frequency Collective Modes in Dry and Hydrated Proteins. Biophys. J. 1989, 56, 713-716.

(183) Conti Nibali, V.; D'Angelo, G.; Paciaroni, A.; Tobias, D. J.; Tarek, M. On the Coupling Between the Collective Dynamics of Proteins and their Hydration Water. J. Phys. Chem. Lett. 2014, 5, 11811186.

(184) Orecchini, A.; Sebastiani, F.; Jasnin, M.; Paciaroni, A.; De Francesco, A.; Petrillo, C.; Moulin, M.; Haertlein, M.; Zaccai, G.; Sacchetti, F. Collective Dynamics of Intracellular Water in Living Cells. J. Phys.: Conf. Ser. 2012, 340, 012091.

(185) Longo, M.; Marconi, M.; Orecchini, A.; Petrillo, C.; Monaco, G.; Calvitti, M.; Pirisinu, I.; Romani, R.; Sacchetti, F.; Sebastiani, F.; et al. Terahertz Dynamics in Human Cells and Their Chromatin. J. Phys. Chem. Lett. 2014, 5, 2177-2181.

(186) Rahman, A.; Stillinger, F. H. Propagation of Sound in Water. A Molecular-Dynamics Study. Phys. Rev. A: At., Mol., Opt. Phys. 1974, 10, 368 .

(187) Pontecorvo, E.; Krisch, M.; Cunsolo, A.; Monaco, G.; Mermet, A.; Verbeni, R.; Sette, F.; Ruocco, G. High-Frequency Longitudinal and Transverse Dynamics in Water. Phys. Rev. E 2005, 71, 011501.
(188) Cimatoribus, A.; Saccani, S.; Bencivenga, F.; Gessini, A.; Izzo, M.; Masciovecchio, C. The Mixed Longitudinal-Transverse Nature of Collective Modes in Water. New J. Phys. 2010, 12, 053008.

(189) Cunsolo, A.; Kodituwakku, C. N.; Bencivenga, F.; Frontzek, M.; Leu, B. M.; Said, A. H. Transverse Dynamics of Water Across the Melting Point: A Parallel Neutron and X-ray Inelastic Scattering Study. Phys. Rev. B: Condens. Matter Mater. Phys. 2012, 85, 174305.

(190) Sampoli, M.; Ruocco, G.; Sette, F. Mixing of Longitudinal and Transverse Dynamics in Liquid Water. Phys. Rev. Lett. 1997, 79, 16781681.

(191) Temleitner, L.; Stunault, A.; Cuello, G. J.; Pusztai, L. Neutron Diffraction of Hydrogenous Materials: Measuring Incoherent and Coherent Intensities Separately. Phys. Rev. B: Condens. Matter Mater. Phys. 2015, 92, 014201.

(192) Gaspar, A. M.; Busch, S.; Appavou, M. S.; Haeussler, W.; Georgii, R.; Su, Y. X.; Doster, W. Using Polarization Analysis to Separate the Coherent and Incoherent Scattering from Protein Samples. Biochim. Biophys. Acta, Proteins Proteomics 2010, 1804, 76-82.

(193) Wochner, P.; Gutt, C.; Autenrieth, T.; Demmer, T.; Bugaev, V.; Ortiz, A. D.; Duri, A.; Zontone, F.; Grubel, G.; Dosch, H. X-Ray Cross Correlation Analysis Uncovers Hidden Local Symmetries in Disordered Matter. Proc. Natl. Acad. Sci. U. S. A. 2009, 106, 11511-11514.

(194) Palmer, J. C.; Martelli, F.; Liu, Y.; Car, R.; Panagiotopoulos, A. Z.; Debenedetti, P. G. Metastable Liquid-Liquid Transition in a Molecular Model of Water. Nature 2014, 510, 385-388.

(195) Limmer, D. T.; Chandler, D. Theory of Amorphous Ices. Proc. Natl. Acad. Sci. U. S. A. 2014, 111, 9413-9418.

(196) Paesani, F.; Iuchi, S.; Voth, G. A. Quantum Effects in Liquid Water from an $\mathrm{Ab}$ Initio-based Polarizable Force Field. J. Chem. Phys. 2007, 127, 074506

(197) Grubel, G.; Stephenson, G. B.; Gutt, C.; Sinn, H.; Tschentscher, T. XPCS at the European X-ray Free Electron Laser Facility. Nucl. Instrum. Methods Phys. Res., Sect. B 2007, 262, 357-367.

(198) Lehmkuhler, F.; Gutt, C.; Fischer, B.; Schroer, M. A.; Sikorski, M.; Song, S.; Roseker, W.; Glownia, J.; Chollet, M.; Nelson, S.; et al. Single Shot Coherence Properties of the Free-Electron Laser SACLA in the Hard X-ray Regime. Sci. Rep. 2014, 4, 5234. 